\title{
Versandapotheken in Deutschland - Die Geburt einer neuen Dienstleistung
} Wer wird eigentlich der Vater?

\author{
Von Martin Gersch
}

In Folge aktueller und zukünftiger Reformen des Gesundheitswesens (u. a. GMG 2004) kommt es erstmalig zur Gründung so genannter Versand- und Internetapotheken in Deutschland, die gegenüber dem traditionellen Leistungsangebot stationärer Apotheken durchaus innovative Services realisieren. Auf der Basis des Resource-based View analysiert der Beitrag mögliche Träger eines ECommerce-Geschäftssystems. Nach der Darstellung notwendiger Partialmodelle eines Geschäftssystems Versandapotheke werden die kritischen Erfolgsfaktoren identifiziert. Hierfür notwendige und bei denkbaren Trägern des Geschäftssystems vorhandene Ressourcen und Kompetenzen werden analysiert. Da kein Akteur über alle notwendigen Eigenschaften verfügt, folgt eine Diskussion grundsätzlicher Alternativen des Kompetenzund Ressourcenerwerbs. In Abhängigkeit von Eckpunkten einer zukünftigen Marktregulierung kann insbesondere - neben den auf absehbare Zeit als unverzichtbar erscheinenden Apothekern - der deutsche, gegebenenfalls aber auch der international agierende Pharmagroßhandel als möglicher „Vater" zukünftiger Service-Angebote in innovativen Geschäftssystemen identifiziert werden.

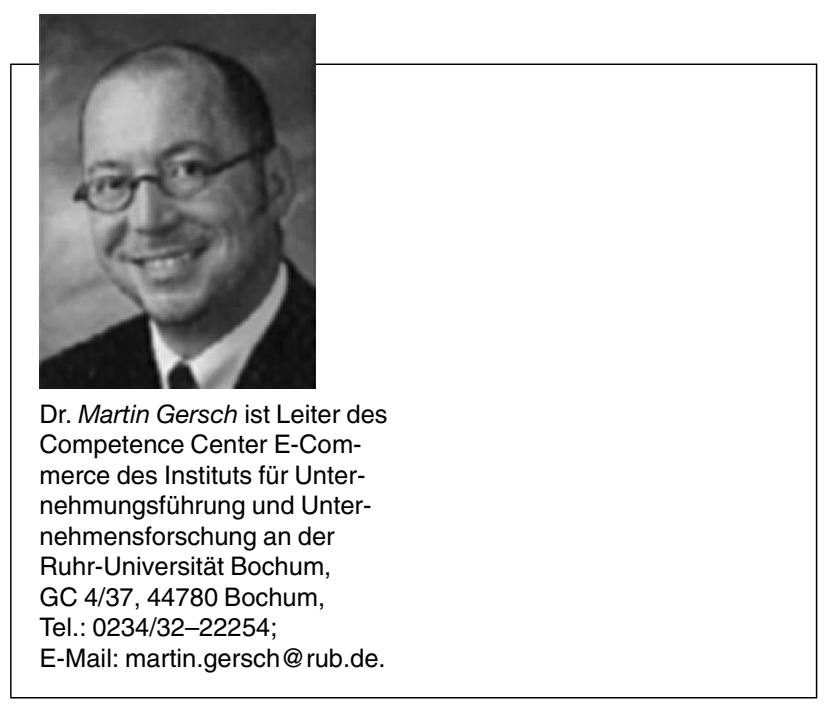

\section{Innovative Geschäftssysteme zur Beherr- schung neuer Erfolgsfaktoren in veränderten ökonomischen Rahmenbedingungen}

\subsection{Die Etablierung neuer E-Commerce- Geschäftssysteme}

E-Commerce und E-Business sind Schlagworte einer so genannten „Internetökonomie“ [1]. Leistungserstellung, Koordination und Transaktionen haben durch Digitalisierung und Vernetzung veränderte oder gar neue Regeln bekommen, innerhalb derer die ,alten“ Restriktionen und Begrenzungen scheinbar nicht mehr in gewohnter Weise gelten (Schmid 2001; Shapiro/Varian 1998; Zerdick et al. 2001).

Häufig sind neue technische Möglichkeiten aber nur der Auslöser für eine fundamentale Veränderung traditioneller Branchenstrukturen und Wettbewerbsregeln. Die generischen Treiber der Veränderungen sind z. B. in Deregulierung, ineffizienten Wertketten oder wenig nachfrageorientierten Leistungsangeboten in den bisherigen Marktstrukturen zu sehen. Internetgestützte Geschäftssysteme treten in Konkurrenz zu bereits etablierten Angeboten auf traditionellen Absatzmärkten oder erfordern durch innovative Leistungsangebote sogar die Abgrenzung neuer Branchen- und Marktgrenzen. Dies wird auch zur Geburtsstunde neuer Dienstleistungsangebote, die bisher in den etablierten Märkten nicht realisiert wurden. Voraussetzung für ein erfolgreiches Angebot ist die Beherrschung der Kritischen Erfolgsfaktoren entsprechender Geschäftssysteme. Notwendige Kompetenzen besitzen aber nicht nur etablierte Anbieter aus vermeintlich verwandten Branchen. Mögliche Geschäftssystembetreiber stammen eventuell aus ganz unterschiedlichen Märkten. Sie werden jetzt zu potenziellen Konkurrenten. Insbesondere die Notwendigkeit zur kooperativen Realisierung entsprechender Leistungsangebote durch bisherige Geschäftspartner und/oder potenzielle Wettbewerber wird nachfolgend anhand eines aktuellen Praxisbeispiels aus dem Dienstleistungsbereich ausführlicher betrachtet [2].

Im ersten Schritt werden in Abschnitt 1.2 unter Verwendung eines Partialmodells Eckpunkte neuer Geschäftssysteme und innovativer Dienstleistungsangebote skizziert. Der Resource-based View wird in Kapitel 2 zur Identifikation notwendiger und bei denkbaren Akteuren vorhandener Ressourcen und Kompetenzen genutzt. $\mathrm{Ka}$ pitel 3 diskutiert auf dieser Grundlage geeignet erschei- 


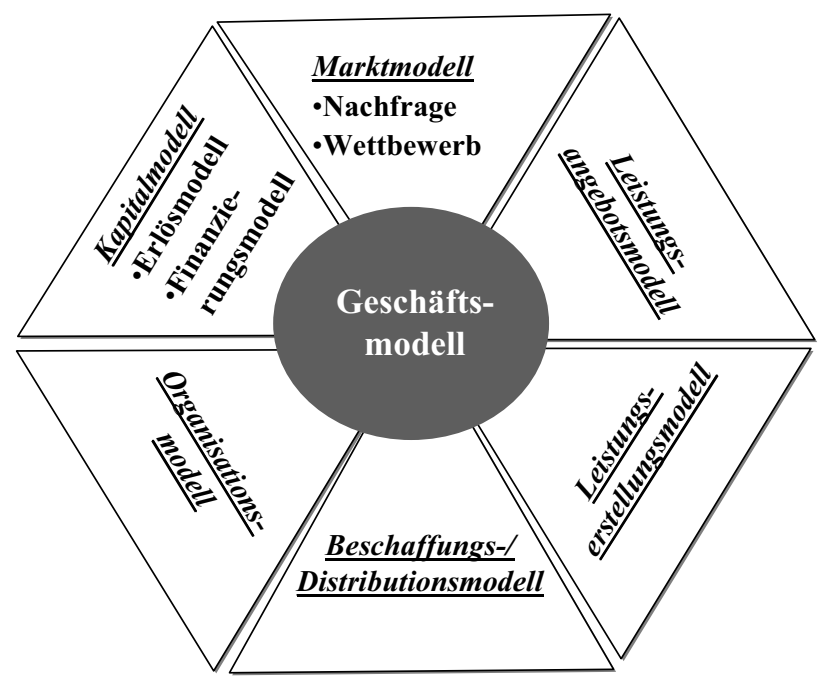

Abb. 1: Relevante Teilbereiche eines Geschäftsmodells [6]

nende Kooperationsformen, da keiner der potenziellen Geschäftssystemträger über alle notwendigen Voraussetzungen verfügt. Der Ausblick in Kapitel 4 wagt eine Prognose bezüglich der im Titel dieses Beitrages gestellten „Vaterschaftsfrage“. Neben den zu erfüllenden Mindestvoraussetzungen hinsichtlich benötigter Ressourcen und Kompetenzen sind hierbei weitere strategische Faktoren zu beachten, die einen nachhaltigen Erfolg der neuen Dienstleistungsangebote beeinflussen. Je nach Erscheinungsform zukünftiger Versandapotheken handelt es sich um eine klassische Dis- oder Reintermediation traditioneller Wertschöpfungsstrukturen.

\subsection{Versandapotheken in Deutschland als Beispiel internetgestützter E-Commerce- Geschäftssysteme}

Vor dem Hintergrund der kontinuierlich steigenden Kosten im Gesundheitswesen und den damit verbundenen Forderungen nach Reformen findet aktuell eine lebhafte, z. T. stark emotional geführte Diskussion um neue Geschäftssysteme im Arzneimittelsektor statt. Vor allem die Legalisierung auch internetgestützter Geschäftssysteme [3] „Versandapotheke“ [4] steht bei dieser Diskussion im Mittelpunkt. Obwohl E-Commerce in vorgelagerten Wertschöpfungsstufen in Deutschland (zwischen den 16 deutschen Pharmagroßhändlern und den derzeit ca. 21.500 öffentlichen Apotheken) seit den 1970er Jahren (Gersch 1998, S. 419 ff.) und im Endkundengeschäft international (Badenhoop/Seiter/Emrich 2002) seit mehreren Jahren bereits Realität ist, besteht in Deutschland für apothekenpflichtige Produkte im EndkonsumentenBereich bis Ende 2003 noch ein Versandhandelsverbot. Aufgrund der aktuellen Gesundheitsreform 2003/2004 [5], anstehender Urteile des Europäischen Gerichtshofes und auch von Erfahrungen in anderen Ländern ist aber in Zukunft mit deutlichen Veränderungen in Deutschland zu rechnen (u. a. BMG 2003; Pfaff et al. 2002; Prinz/Vogel 2002; Riehm et al. 2002). Die Frage ist kaum noch ob, sondern eher wie die neuen medikamentenbezogenen

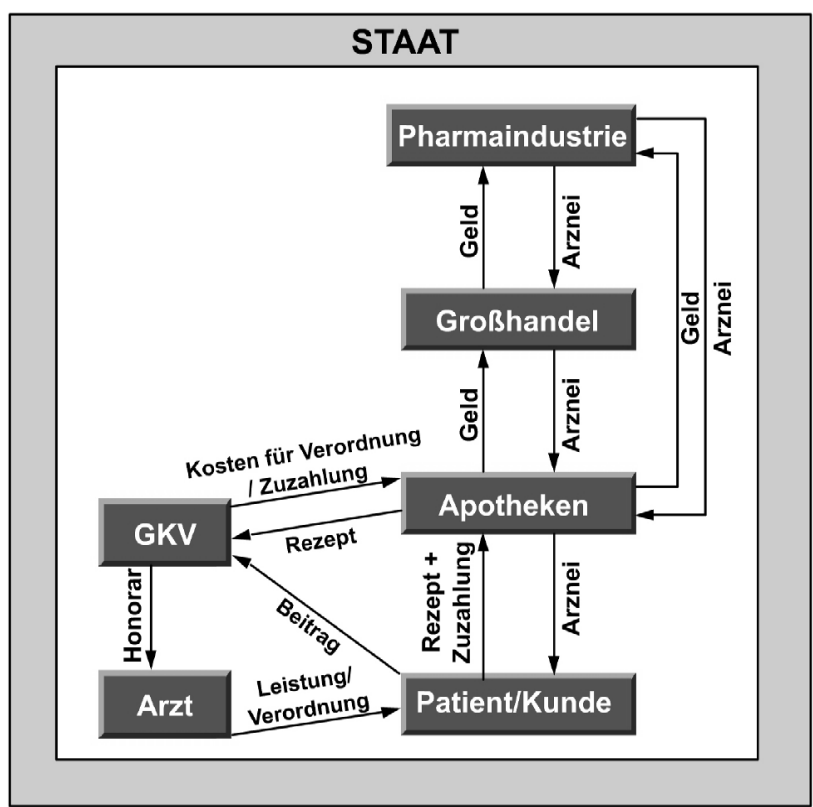

Abb. 2: Die traditionelle Struktur des deutschen Pharmamarktes (Quelle: Kunz, 2001, S. 64)

Services ausgestaltet werden und vor allem, welche Akteure die Erfolgsfaktoren der neuen Geschäftssysteme beherrschen.

Die Darstellung erfolgt hierbei in Anlehnung an die weiter entwickelte Systematik von Wirtz (siehe Abb. 1; Gersch 2002, S. 412 f.).

\subsubsection{Apotheken als Bezugspunkt des Marktmodells}

Das Marktmodell analysiert die für das Geschäftssystem relevanten Märkte. Ausgangspunkt der Überlegungen ist hier der traditionelle Arzneimittelmarkt in Deutschland und seine vorgelagerten Wertschöpfungsstufen (Abb. 2) [7]. Die Versorgung der Endkunden erfolgte 2002 durch 21.465 öffentliche Apotheken (563 Krankenhausapotheken bleiben nachfolgend unberücksichtigt), die neben apothekenpflichtigen Produkten auch so genannte Ergänzungssortimente sowie eine Reihe von Services, z. B. in Form von Behandlungsleistungen und Beratung, anbieten. Hiermit erzielten sie im Jahre 2002 einen Gesamtumsatz von 31,1 Mrd. Euro (ohne MwSt.) (ABDA 2003).

Die bisherige rechtliche Reglementierung bildet „,vier Säulen" für die traditionelle Position öffentlicher Apotheken (Kerckhoff 2002; Kunz, 2001; Riehm et al. 2002): Das Angebotsmonopol ( $\$ 43$ AMG, $\$ 1$ ApoG) sichert die Exklusivität des Absatzes von Arzneimitteln über Apotheker. Das fixierte Preissystem (§78 AMG; §2-3 AMPreisV) sichert über Höchstzuschläge auf der Großhandels- und Festzuschläge auf der Apothekenstufe bundesweit einheitliche Abgabepreise. Das Versandhandelsverbot ( $\$ 43 \mathrm{AMG} ; \S 17 \mathrm{ApBetrO}$ ) beschränkt die Abgabe auf die Räumlichkeiten einer Apotheke (die so genannte „Botenausnahme“ wird seit Jahren von vielen Apotheken überstrapaziert, so dass Versandapotheken de 
facto schon länger existieren). Und schließlich verhindert das Fremd- und Mehrbesitzverbot die Bildung so genannter Apothekenketten ( $\$ 7$ ApoG), da jeder Apotheker selbständig sein muss und auch nur eine Apotheke betreiben darf. Daneben gilt eine Reihe weiterer Regulierungen, die die Aktionsfreiheit der Akteure beschränken, so z. B. das Werbeverbot oder die Beschränkung des Sortiments auf ein apothekenübliches Waren- und Leistungsangebot (Riehm et al. 2002, S. 157 ff.; Sterzel/Wassener 2001; Prinz/Vogel 2002).

Eine weitere Besonderheit besteht in der marktunüblichen Trennung zwischen Entscheider (Arzt), Kostenträger (Krankenkasse) und Verwender (Patient). Durch diese Konstruktion fehlen bisher weitestgehend wirksame Anreizstrukturen: die Nachfrage nach Arzneimitteln besitzt faktisch keine Preiselastizität, das Angebot wird vordergründig durch gesundheitspolitische Zielsetzungen bestimmt. Auch durch diese Konstruktion begründet, hat sich in Deutschland ein international unüblich breites und tiefes Arzneimittelangebot etabliert. Während andere Länder mit 3.500 (Schweden) bis 14.000 (Großbritannien) zugelassenen Arzneimitteln auskommen, sind in Deutschland im Jahre 2002 über 52.000 verkehrsfähige Arzneimittel auf dem Markt.

Insgesamt hat sich - insbesondere durch die Digitalisierung und Vernetzung der den Apotheken vorgelagerten Wertschöpfungskette - ein fast beispiellos leistungsfähiges Gesamtsystem bei der Arzneimittelversorgung entwickelt. Jede Apotheke kann innerhalb weniger Stunden über 100.000 Produkte für den Patienten bereitstellen. Der Pharmagroßhandel übernimmt durch seine Dienstleistungen die Ausgleichsfunktion, da Apotheken üblicherweise nur 3.000 bis 8.000 der gängigsten Präparate vorrätig halten. Bis zu fünfmal am Tag werden Apotheken durch den Großhandel mit einer Defektquote von unter einem Prozent und einem Lieferfenster von ca. 2-3 Stunden versorgt. $93 \%$ der Bestellungen zwischen Apotheken und Großhandel laufen hierbei online, noch über $70 \%$ der Bestellungen des Großhandels bei den Pharmaherstellern sind seit den 1970er Jahren als E-Commerce anzusehen (Riehm et al. 2002, S. 164). Somit gehört diese Branche zu den Bereichen mit dem höchsten Diffusionsgrad derartiger Anwendungen (Gersch 2001).

Dass zukünftig Versandapotheken diese ,elektronische Kette" bis zum Endkunden schließen, deutet sich aufgrund der Gesundheitsreform 2003/2004 und aktueller Diskussionen an [5]. So zeigen Modellrechnungen und internationale Vergleiche das mögliche Einsparpotenzial, welches auch von politischer Seite als realistischer und erstrebenswerter Beitrag zur Senkung der Kosten im Gesundheitswesen gesehen und akzeptiert wird (Pfaff et al. 2002; Prinz/Vogel 2002). Weitere einflussreiche Interessengruppen unterstützen aktiv die Etablierung innovativer Geschäftssysteme. Neben politischen Entscheidungsträgern sind dies insbesondere Krankenkassen, die u. a. auch eine bessere und effizientere Betreuungsmöglichkeit verschiedener Patientengruppen, z. B. durch innova- tive Service-Angebote im Rahmen so genannter „Disease Management Konzepte“ [8], anstreben. Auch Pharmahersteller sehen in Versandapotheken eine Chance zur Disintermediation bisheriger Wertkettenstrukturen und zur effizienten „Direktbehandlung“ interessanter Patientengruppen mit deutlich geringeren Streuverlusten z. B. im Bereich Information und Kommunikation. Dem steht insbesondere die Gruppe der Apotheker entgegen, die um ihre wirtschaftliche Situation, aber insbesondere auch um ihre Unabhängigkeit fürchten (Gersdorff 2002; Mihm 2002; www.pro-apotheke.de).

\subsubsection{Mögliche Leistungsangebotsmodelle}

Die aktuelle Diskussion zeigt ein relativ deutliches Bild bezüglich der zukünftigen Ausgestaltung des Geschäftssystems Versandapotheke. Aufgrund der überragenden Bedeutung einer staatlich kontrollierten Versorgungssicherheit werden ausländische Anbieter, die nicht den europäischen bzw. deutschen Bestimmungen unterliegen, möglichst vom Markt ausgeschlossen. Nur über das Internet agierende Anbieter außerhalb der Europäischen Union werden daher kaum eine Chance erhalten. Vielmehr zeichnen sich die im April 2002 durch einen so genannten „runden Tisch“ im Bundesgesundheitsministerium erarbeiteten und im Rahmen der Gesundheitsreform 2003/2004 weitgehend umgesetzten Eckpunkte als realistische Rahmenbedingungen zukünftiger Leistungsangebote ab (BMG 2002b; 2003). Versandapotheken sind Apotheken mit besonderem Vertriebsweg, neuen und z. T. patientengruppenspezifischen oder gar individuellen Zusatzdienstleistungen sowie tendenziell geringeren Kosten, die - falls rechtlich ermöglicht - in geringeren Verkaufspreisen oder Rabatten an die Endkunden bzw. Kostenträger (insb. Krankenkassen) weitergegeben werden können. Sie müssen ebenso wie traditionelle Apotheken u. a. ein Vollsortiment anbieten, Notdienste versehen, die sachgemäße Betreuung durch Fachpersonal gewährleisten (inkl. Apotheker als Verantwortlicher) und darüber hinaus sicherstellen, dass versandte Arzneimittel nur an den Patienten mit Rezept (oder Bevollmächtigten) ausgehändigt werden.

Ihr Angebot ist nicht grundsätzlich für alle bisherigen Apothekenkunden überlegen attraktiv (z. B. eilige Arzneimittel bei akutem Bedarf oder Wunsch nach persönlichem Kontakt und Beratung), daher droht keine vollständige Substitution stationärer Apotheken. Allerdings sind Erfolge bei besonders geeigneten Zielgruppen sehr wahrscheinlich. Hierzu zählen insbesondere chronisch Kranke mit einem vorhersehbaren und konstanten Arzneimittelbedarf, immobile Kunden ohne akuten Bedarf, aber auch Patienten mit einem sehr speziellen Behandlungs- und Informationsbedarf. Insgesamt schätzen unabhängige Studien und Experten einen realisierbaren Marktanteil zwischen 8 und $15 \%$ des Gesamtmarktes (mit deutlichen Unterschieden bei einzelnen Produktund Zielgruppen) (BMG 2002a; Kerckhoff 2002; Pfaff et al. 2002; Prinz/Vogel 2002). 
Es ist daher zu erwarten, dass sich viele Versandapotheken in ihrem Angebot - trotz Vollsortimentspflicht - auf einzelne Regionen, Patientengruppen und/oder Medikationsbereiche bzw. (in Kooperation mit Pharmaherstellern) Einzelprodukte spezialisieren. Diese Spezialisierung wird sich insbesondere in den zusätzlichen, z. T. innovativen (Electronic) Services äußern, die hierbei vollumfänglich auch Online-Medien zur Information, Interaktion und Community-Bildung einbeziehen werden (Däinghaus 2003, S. 99 ff.) [9]. Neben diesen spezialisierten Leistungsangeboten gehen aktuelle Studien auch von der Herausbildung großer, besonders effizient und kostengünstig agierender Versandapotheken aus, die mit einem geschätzten Umsatz ab ca. 250 Mio. Euro die GröBe traditioneller stationärer Apotheken (2002 betrug der Durchschnittsumsatz 1,44 Mio. Euro) um mehr als den Faktor 170 übersteigen würden (ABDA 2003; Pfaff et al. 2002, S. 36 ff.).

\subsubsection{Notwendige Wertkettenaktivitäten im Leis- tungserstellungs-, Beschaffungs- und Distributions-} modell

Für das Angebot der beschriebenen Leistungen muss das in $A b b .3$ skizzierte Leistungserstellungsmodell so oder in ähnlicher Form realisiert werden. Neben den primären
Aktivitäten, wie z. B. Bestellannahme, Kommissionierung der Einzellieferung oder Einkauf und Transport der angebotenen Ware, bedarf es einer Reihe sekundärer Aktivitäten, die auf der linken Seite der Abbildung genannt werden. Eine besondere Bedeutung wird die Möglichkeit eines „elektronischen Rezeptes“ erhalten. Versandapotheken müssen im Rahmen der traditionellen Strukturen den Versand, die Überprüfung und die Digitalisierung z. T. noch von Hand erstellter Rezepte realisieren (siehe hierzu das Schweizer Geschäftssystem Mediservice: www.mediservice.ch). Durch derzeit diskutierte Möglichkeiten elektronisch erstellter und übermittelter Rezepte würden nicht nur diese Medienbrüche entfallen und Kosten eingespart, der ausstellende Arzt würde - je nach Ausgestaltung des elektronischen Rezeptes - vermutlich auch eine andere Bedeutung bei der Auswahl des zu nutzenden Apotheken-Geschäftssystems erhalten.

\subsubsection{Das Kapitalmodell des Geschäftssystems Ver- sandapotheke}

Nach Angaben der $A B D A$ konnten die traditionellen Apotheken in den letzten Jahren trotz sinkender Handelsspannen ein positives ökonomisches Ergebnis realisieren. 2002 erreichten sie im Durchschnitt aller Apotheken ein Betriebsergebnis nach Vollkosten (u. a. inkl. kalkula-

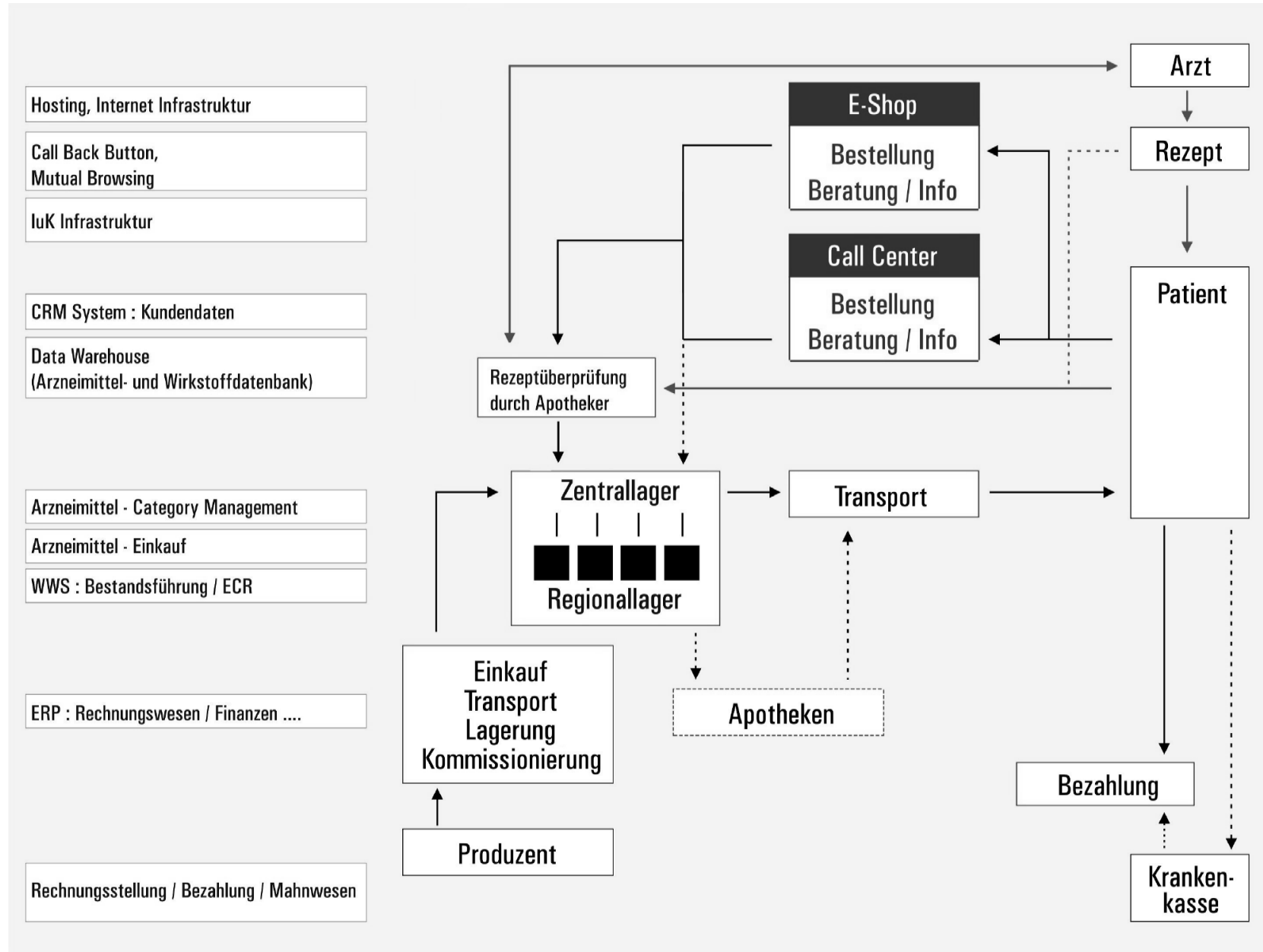

Abb. 3: Notwendige Wertkettenaktivitäten des Geschäftssystems Versandapotheke (Quelle: Gersch/Theißen 2003, S. 30) 
torischer Kosten!) von noch $0,5 \%$ des Umsatzes nach $1,4 \%$ im Vorjahr (ABDA 2003).

Modellrechnungen gehen bei Versandapotheken - je nach konkreter Ausgestaltung der rechtlichen Rahmenbedingungen, je nach Ausrichtung des Leistungsangebotes und je nach Betriebsgröße - von einem realisierbaren Betriebsergebnis zwischen $6 \%$ und $11 \%$ des Umsatzes aus (Pfaff et al. 2002, S. 36 ff.; Berechnungen erfolgten noch ohne das so genannte "Vorschaltgesetz" vom November 2002 und ohne die Gesundheitsreform 2003/ 2004 [5]). Dies sind im Vergleich zu anderen Bereichen des Einzelhandels überragende Ergebnisse. Basieren die Studien und Modellrechnungen auch auf einzelnen, z. T. diskussionswürdigen Prämissen, so zeigt sich insgesamt dennoch die grundsätzlich positiv beurteilte Zukunft des Geschäftssystems Versandapotheke. Typisch für E-Commerce-Geschäftssysteme werden auf der Erlösseite nicht nur direkte Leistungsentgelte realisiert. Daneben bestimmen weitere Erlösquellen - z. B. Werbeeinnahmen, Vermarktungsverträge mit der Pharmaindustrie oder Informationsdienstleistungen bzgl. einzelner Arzneimittel oder Patientengruppen - den Erfolg des Geschäftssystems.

\section{Identifikation Kritischer Erfolgsfaktoren und notwendiger Kernkompetenzen}

Die ganzheitliche Sicht eines E-Commerce-Geschäftssystems ermöglicht die Identifikation wesentlicher Einflussfaktoren auf die zukünftige Kosten- und Erlösstruktur des Geschäftssystems. Hierdurch lassen sich Kritische Erfolgsfaktoren benennen, die über den zukünftigen Erfolg oder Misserfolg entscheiden. In einem zweiten Schritt müssen dann notwendige Kompetenzen identifiziert werden, die die Beherrschung der Erfolgsfaktoren sicherstellen. Abschließend sind unterschiedliche Quellen des Kompetenzerwerbs zu identifizieren und gegeneinander abzuwägen. Kooperationen erscheinen bei der Sicherung notwendiger Ressourcen und Kompetenzen als realistische Alternative zu Eigenerstellung, Fremderwerb oder Fusion (Backhaus 2003, S. 282).

\subsection{Der Zusammenhang zwischen Kritischen Erfolgsfaktoren sowie notwendigen und verfüg- baren Kernkompetenzen aus der Perspektive des Resource-based View}

Jedes E-Commerce-Geschäftssystem zur Realisierung z. T. integrativ erstellter Leistungsangebote ist durch spezielle Erfolgsfaktoren gekennzeichnet, deren Beherrschung erfolgreiche von nicht erfolgreichen Ansätzen unterscheidet. In der Terminologie des Resource-based View (RBV) versetzen so genannte Ressourcen einen Anbieter in die Lage, diesen Anforderungen gerecht zu werden (Bamberger/Wrona 1996a; Barney 1991; Freiling 2001; 2002; Gersch 2002). Jedes Unternehmen kann demnach als spezifische Kombination von Ressourcen interpretiert werden, die im Zusammenspiel darüber entscheiden, ob das Unternehmen auf seinen relevanten Märkten nachhaltige Wettbewerbsvorteile realisieren kann. Als Ressourcen werden sämtliche zur Leistungserstellung verwendeten Potenzial- und Inputfaktoren interpretiert, die Leistungserstellungsprozesse und Absatzobjekte ermöglichen und die insbesondere durch Veredelungsprozesse zu unternehmenseigenen Merkmalen für Wettbewerbsfähigkeit weiterentwickelt wurden (Freiling 2001, S. 84 ff.). Ressourcen können sowohl einzelne (,veredelte“ = so nicht am Markt erhältliche) Faktoren sein, als auch bereits zueinander in spezifischer Beziehung stehende Kombinationen und Netzwerke von Faktoren. Ressourcen im Sinne des RBV werden somit $\mathrm{zu}$ einer besonderen Teilmenge der Potenzialdimension des oder der Geschäftssystemträger(s). Sie sichern die Wettbewerbsfähigkeit und sind gleichzeitig durch so genannte Isolationselemente und -mechanismen vor Dritten geschützt. Ressourcen sind das Ergebnis unternehmensspezifischer „Veredelungsprozesse“ generischer, durchaus marktfähiger Inputfaktoren (so auch Schneider 1998). Abnutzbarkeit, Transferierbarkeit, Imitierbarkeit und Substituierbarkeit werden zu zentralen Ressourceneigenschaften, deren Ausprägung über Art und Ausmaß möglicher Wettbewerbsvorteile entscheidet (Bamberger/Wrona 1996a, S. 135 ff. und 1996b, S. 387; Freiling 2001, S. 94 ff.).

Die Verfügung über Ressourcen in einem Zeitraum/-punkt begründet als ein wichtiges Element die Möglichkeit eines Unternehmens, die als kritisch identifizierten Erfolgsfaktoren zu beherrschen und somit Erfolg oder Misserfolg mit einem Geschäftssystem zu haben. Allein die Verfügungsgewalt über Ressourcen reicht aber als Erklärung nicht aus. Unternehmen müssen auch die Kompetenz besitzen, diese Ressourcen im Bedarfsfall geeignet $\mathrm{zu}$ aktivieren und in wettbewerbsfähige Leistungsangebote umzusetzen (Schneider 1998. S. 345). (Kern-)Kompetenzen werden somit zur „Eigenschaft" eines Unternehmens, vorhandene Ressourcen und personelle Fähigkeiten ,richtig“ zu nutzen.

Die Analyse wird somit folgerichtig mit der Skizzierung Kritischer Erfolgsfaktoren fortgesetzt. Diese werden als zentrale Anforderungen identifiziert, welche im Rahmen eines konkreten Geschäftssystems zu erfüllen sind, damit die Aktivität grundsätzlich erfolgreich sein kann. In einem Folgeschritt gilt es zu analysieren, welche Ressourcen und Kompetenzen notwendig erscheinen, um die Erfolgsfaktoren $\mathrm{zu}$ beherrschen. Hierbei ist dieser $\mathrm{Zu}$ sammenhang indeterminiert (Bamberger/Wrona 1996a, S. 138; Gersch 2002, S. 419). D. h., verschiedene Kombinationen unterschiedlicher Potenzialfaktoren können prinzipiell bewirken, dass ein Anbieter die jeweils als kritisch identifizierten Erfolgsfaktoren beherrscht. Allenfalls tendenziell lassen sich zentrale Ressourcen benennen, die als unverzichtbar erscheinen. Es lässt sich derart ein (nicht allein richtiges) Soll-Profil ableiten, über welche Ressourcen und Kompetenzen ein Geschäftssystemträger verfügen sollte. Diesem Soll-Profil können Ist- 
Profile von Anbietern gegenübergestellt werden. Es ergeben sich Übereinstimmungen, aber voraussichtlich auch Lücken, die es zu füllen gilt.

\subsection{Kritische Erfolgsfaktoren der Versand- apotheke und hierfür notwendige Kompetenzen}

Für jeden denkbaren Träger eines möglichen Geschäftssystems Versandapotheke gilt es, die nachfolgenden drei Erfolgsfaktoren als Grundvoraussetzungen zu erfüllen [10]. Die zunächst allgemein klingenden Aspekte müssen dabei insbesondere unter Nutzung neuer technischer Möglichkeiten im Rahmen des Leistungsangebotes und der Leistungserstellung konsequent beachtet werden. Nachfolgend werden gleichzeitig die jeweils erforderlich erscheinenden Ressourcen und Kompetenzen skizziert:

\section{- Vertrauen:}

Arzneimittel sind Produkte mit weitgehenden Vertrauenseigenschaften. Notwendiges Vertrauen in einen Anbieter bezieht sich hierbei sowohl auf die Qualität der Produkte und der Beratung als auch auf die Verlässlichkeit im Notfall und seine Verschwiegenheit bezüglich der erlangten kundenbezogenen Informationen. Zur Sicherstellung dieses Vertrauens gilt es, die Zuverlässigkeit sämtlicher kritischen Prozesse des Geschäftssystems zu gewährleisten sowie auch extern nachzuweisen und glaubwürdig zu kommunizieren. Neben gesetzlichen Zulassungs- und Überwachungsverfahren bieten sich insbesondere etablierte Marken, qualifizierte Zertifizierungen und/ oder Gütesiegel an.

\section{- Erfolgsorientierte Sortimentsgestaltung:}

Dies bedeutet insbesondere Konzentration auf attraktive Betätigungsfelder trotz angebotenem Vollsortiment, zu dem auch Versandapotheken zukünftig gesetzlich verpflichtet sein werden (BMG 2003). Dies könnten insbesondere spezielle Indikationsgruppen sein (z. B. chronisch Kranke mit hohem permanentem Medikamentenbedarf und hohem Warenwert je Lieferung), Direktabsatzkonzepte mit einzelnen Pharmaherstellern an spezifische Patientengruppen oder die Integration in so genannte „Disease Management Konzepte“" [8] freier Dienstleister oder in Kooperation mit Krankenkassen zur ganzheitlichen Betreuung selektierter Patientengruppen. Bei all diesen Möglichkeiten kommt es, neben der entsprechenden medizinischen Fachkenntnis, auf zielgruppenspezifische, z. T. sogar patientenindividuelle Leistungsangebotskonzepte an. Hierbei gilt es, das Warensortiment durch geeignete Zusatz- und Ergänzungsleistungen attraktiv zu gestalten. Neben entsprechendem Category Management Know-how sind hierfür One-to-One-Marketing Ansätze gegenüber den Endkunden und Kooperationsmanagement gegenüber möglichen Partnern erforderlich. Im Rahmen von so genannten „Make-or-buy-Entscheidungen“ werden sich die Geschäftssystemträger insbesondere auch überlegen, ob sie geeignete (E-)Services selbst erstellen oder besser
Elemente spezialisierter Unternehmen in ihr Leistungsangebot integrieren [10].

- Effizienz in primären und sekundären Aktivitäten: Trotz der aktuell noch scheinbar positiven ökonomischen Branchensituation ist in Zukunft ein härterer Wettbewerb, z. T. auch über das Instrument Preis (Marge) zu erwarten. Hierbei setzen die Leistungseckdaten der bisherigen mehrstufigen Wertkette zur Arzneimittelversorgung hohe Anforderungen an einen zukünftigen Versandapotheker. Höchste Effizienz im Rahmen aller Prozesse der Leistungserstellung ist ebenso unabdingbar wie wettbewerbsfähige Einkaufspreise, die zukünftig entweder über Volumeneffekte und/oder Exklusivabkommen mit einzelnen Produzenten zu erreichen sind. Insbesondere das Bestandsmanagement durch eine optimierte Bestellpolitik sowie die effizienzorientierte Gestaltung der gesamten logistischen Lieferkette von den Produzenten über die Kommissionierung bis über die so genannte ,letzte Meile“ zum Endkunden kennzeichnen erforderliche Kompetenzen. Hierfür ist die Nutzung unternehmensübergreifender ganzheitlich integrierter IT-Systeme erforderlich. Vorbild ist hierbei das Beispiel des deutschen Pharmagroßhandels (Gersch 1998, S. 437 ff.). Weiterhin muss der Kundenkontakt über mehrere Kontaktkanäle (insbesondere Internet und diverse $\mathrm{Zu}$ gänge über Call-Center) gestaltet werden. Ein besonderes Gewicht muss - trotz des Effizienzdrucks - auf einer zuverlässigen und qualitativ hochwertigen Betreuung durch entsprechend geschulte Call-Center gelegt werden. Die notwendige Integration verschiedener Kontaktkanäle und ggf. Teilleistungen dritter Anbieter in ein geschlossenes und aus Nachfragerperspektive stimmiges Gesamtkonzept mit wettbewerbsfähiger Integralqualität kennzeichnet ganz besondere Herausforderungen zukünftiger Leistungsangebote.

$A b b .4$ fasst die skizzierten notwendigen Ressourcen und Kompetenzen zur erfolgreichen Realisierung des Geschäftssystems Versandapotheke noch einmal übersichtsartig zusammen.

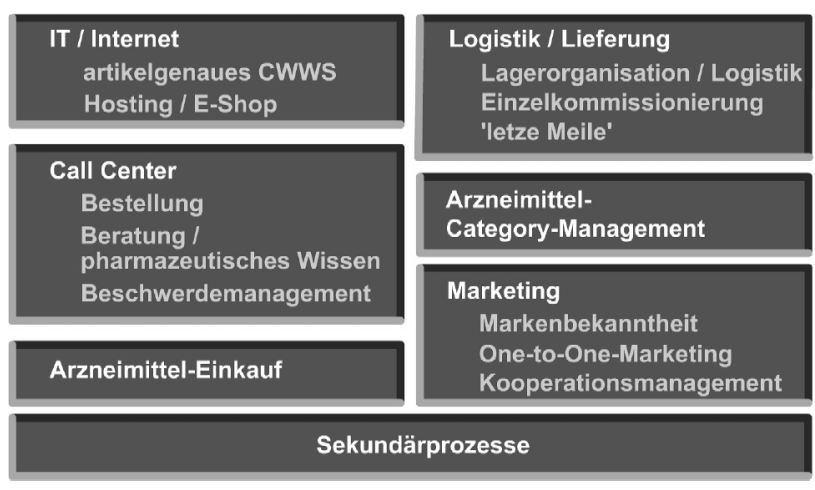

Abb. 4: Notwendige Ressourcen und Kompetenzen zur erfolgreichen Realisierung des Geschäftssystems Versandapotheke (Quelle: Gersch/Theißen 2003, S. 45) 


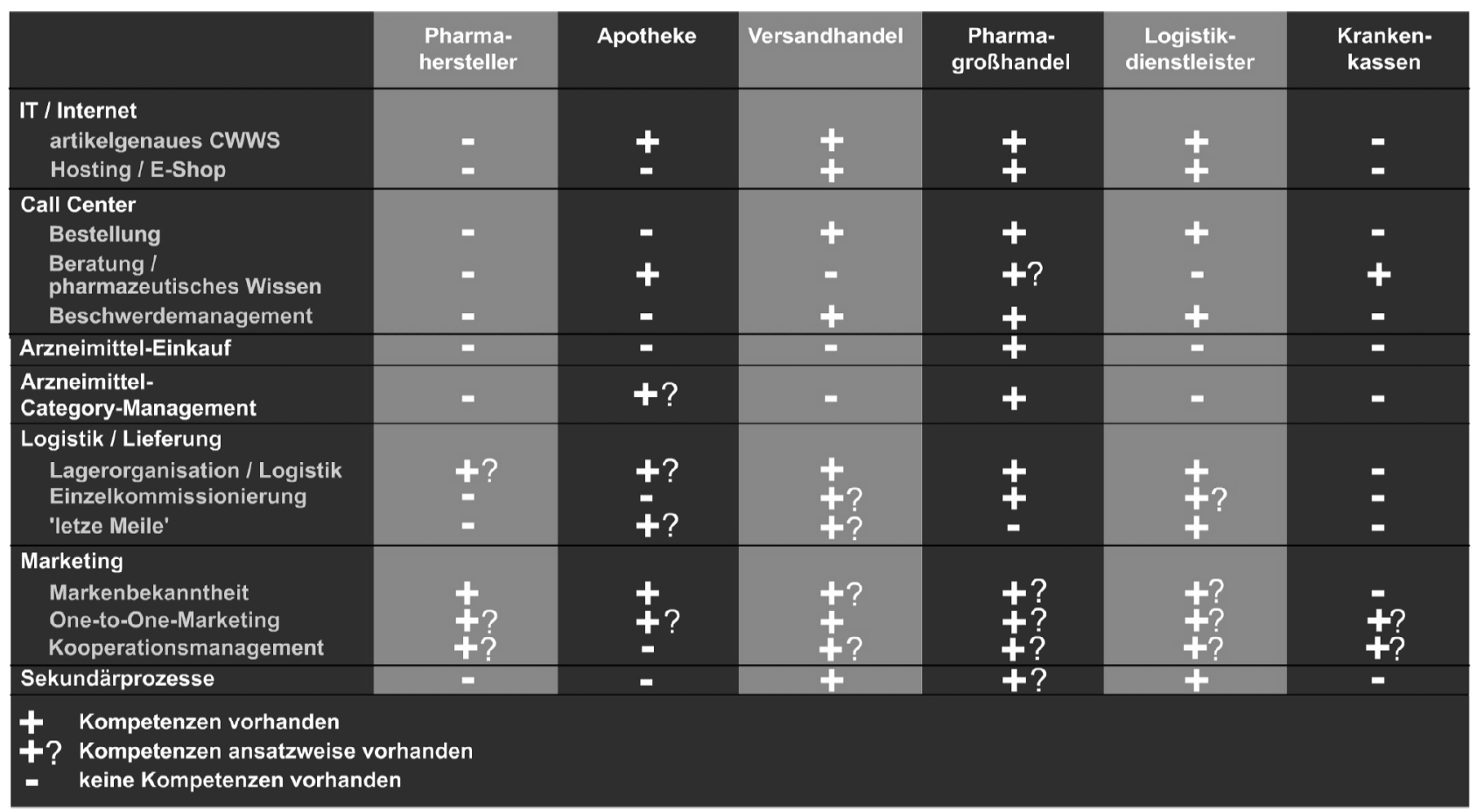

Abb. 5: Denkbare Akteure und ihre verfügbaren Kompetenzen (Quelle: Gersch/Theißen 2003, S. 53)

\subsection{Alternative Akteure zur Realisierung von Versandapotheken und ihre verfügbaren Kompe- tenzen}

Auch Erfahrungen aus anderen Branchen (z. B. Lebensmittelheimbelieferung oder Heimwerkerbedarf) zeigen, dass potenzielle Träger eines Geschäftssystems Versandapotheke nicht nur national und im Umfeld verwandter Branchen zu suchen sind (Gersch 2003). Regelmäßig erweisen sich auch Unternehmen aus anderen Betätigungsfeldern als prinzipiell geeignet. Das Aufbrechen bisheriger deutscher Marktstrukturen kann insbesondere auch das Signal für internationale Akteure sein, ein Engagement auf dem weltweit drittgrößten Markt für Arzneimittel zu prüfen.

Abb. 5 skizziert denkbare Akteure [11]. Bildet die linke Seite ein so genanntes Soll-Profil notwendiger Ressourcen und Kompetenzen, so ergibt sich durch die einzelnen Kompetenzprofile der Akteure insgesamt eine „Kompetenz-Lücken-Analyse“ (Freiling 1998b und 1998c; Gersch 2003). Ohne im Detail die Situation jeden Akteurs darstellen zu können wird deutlich, dass insbesondere der Pharmagroßhandel (PHGH) über die beste Ausgangslage hinsichtlich verfügbarer Ressourcen- und Kompetenzbündel verfügt. Doch auch der PHGH weist Lücken auf, die es zu schließen gilt. So reichen weder die derzeitigen Call-Center Kapazitäten (weder qualitativ noch quantitativ) aus noch verfügt er über die Möglichkeiten zur effizienten Gestaltung der letzten Meile. Insgesamt ist das Angebot endnachfragerorientierter Leistungsbündel inklusive zielgruppenspezifischer oder gar individueller Services in Deutschland eine gänzlich neue Herausforderung für die Grossisten. Die bisher vorwiegend im Business-to-Business-Bereich erworbenen
Kompetenzen im Beziehungs-Marketing gilt es, an das Endkundengeschäft anzupassen. Auch die aktuelle Markenbekanntheit wird für die notwendige Vertrauensposition bei Konsumenten nicht ausreichen [10].

\section{Kooperationen als Instrument des Kompetenz-Managements}

Keiner der in $A b b .5$ skizzierten Akteure scheint allein über alle notwendigen Ressourcen und Kompetenzen für ein wettbewerbsfähiges Geschäftssystem Versandapotheke $\mathrm{zu}$ verfügen. Häufig können fehlende Kapazitäten und Kompetenzen einzelner Akteure nicht schnell genug, nicht ausreichend effizient und nicht in der notwendigen Qualität autonom und unternehmensintern aufgebaut werden (Picot/Reichwald/Wigand 2003, S. 289 ff.; Reiß 2001, $\mathrm{S} .123$ f.). Ein möglicher Zukauf generischer Inputfaktoren mit anschließender Entwicklung zu wettbewerbsrelevanten Ressourcen erscheint aufgrund des großen Zeit- und Kostenbedarfs - zumindest für komplexe Ressourcenbündel - unrealistisch und in Bezug auf die Erfolgsaussichten auch mit großer Unsicherheit behaftet. Allenfalls in einzelnen Kompetenzbereichen eröffnen sich Möglichkeiten zumindest einzelne Ressourcenelemente - gemeinsam mit spezialisierten Dienstleistern zu entwickeln bzw. die Leistung fremd zu vergeben (Backhaus/Meyer 1993, S. 333; Baden-Fuller/Volberda 1998, S. 5; Engelhardt/Reckenfelderbäumer 1993; Zocholl 2003).

Grundsätzlich bieten sich Kooperationen - im Sinne vertraglich geregelter Zusammenarbeit rechtlich selbständiger Unternehmen zur gemeinschaftlichen Erfüllung einer Aufgabe (Picot/Reichwald/Wigand 2003, S. 304) - als denkbares Instrument zur Kombination entsprechender 
Kompetenzbereiche an. Das primäre Kooperationsziel wird die Ergänzung fehlender Ressourcen und Kompetenzen durch andere Partner mit komplementärem Profil sein. Es handelt sich daher überwiegend um so genannte „Closing-Gap-Allianzen“ im Gegensatz zu „CriticalMass-Allianzen“, die bei überlappenden Kompetenzbereichen auf synergetische Mengeneffekte der Zusammenarbeit abstellen (Freiling 1998a, S. 27 ff.; Gersch 2003).

Dies wird aber regelmäßig durch Vorbehalte der beteiligten Akteure und durch so genannte Isolationsmechanismen erschwert, die der Übertragung vorhandener Ressourcen und Kompetenzen in andere Betätigungsfelder entgegenstehen können. Vor einer Analyse dieser zentralen Kooperationsaspekte müssen grundsätzlich denkbare Szenarien der Zusammenarbeit zwischen den möglichen Akteuren identifiziert werden.

\subsection{Denkbare Szenarien bei der Realisierung des Geschäftssystems Versandapotheke}

Unterstellt man in einem ersten Schritt zunächst nur den Wegfall des Versandhandelsverbotes (nach \$43 AMG und $§ 17$ ApBetrO), so erscheinen grundsätzlich folgende sechs Szenarien und Akteurskonstellationen - eventuell auch parallel - möglich [10] [11]:

- Szenario 1: Zukünftiger Alleingang einzelner Pharmagroßhändler (PHGH) unter Nutzung des Angebotes dritter Dienstleistungsanbieter als Spezialisten, z. B. eines Logistikers zur Überwindung der letzten Meile.

- Szenario 2: Kooperative Lösung Apotheke - PHGH. Entweder unter Führung eines Pharmagroßhändlers oder durch Bildung von Großversandapotheken unter Miteinbeziehung eines PHGH.

- Szenario 3: Herausbildung von Großversandapotheken durch Kooperation und Nutzung von Synergien, evtl. durch Koordination eines Dritten (z. B. ABDA, Bundesverband Deutscher Versandapotheker/Innen (BVDVA), MediService aus der Schweiz, Logistiker).

- Szenario 4: Einbruch eines Marktfremden (z. B. internationaler PHGH, nationaler Versandhandel, internationale Versand-/Internetapotheke) in den deutschen Markt.

- Szenario 5: Etablierung vieler kleiner Versandapotheken als zweites Standbein traditioneller Apotheken.

- Szenario 6: Etablierung neuer Services, z. B. Disease Management [8] durch einzelne Krankenkassen, die u. a. Versandapotheken allein oder in Kooperationen realisieren.

Eine deutliche Veränderung erfahren die Szenarien bei der zusätzlichen Aufhebung des Fremd- und Mehrbesitzverbotes (nach $\$ 7$ ApoG). Durch die grundsätzliche Erlaubnis von Apothekenketten würden sich sowohl ausländische Akteure als auch der deutsche Pharmagroßhandel einer grundsätzlich veränderten Situation gegen- übersehen. Bereits jetzt orientieren sich die Großhändler international auf europäischer Ebene. Hierzu gehört auch der Erwerb und die Weiterentwicklung von Apothekenketten in Ländern, in denen dies bereits heute rechtlich möglich ist. So besitzt z. B. die deutsche GEHE 2002 über 1.800 Apotheken u. a. in Großbritannien, Italien, Irland und Norwegen. In Verbindung mit möglichen Disintermediationsgefahren durch große Versandapotheken wäre der Einstieg des Großhandels auch in deutsche Apothekenketten eine realistische Option (Hofmann/ Fröndhoff 2002; Kerckhoff 2002; Ossenberg-Engels 2002). Der Ersatz bisheriger Wertschöpfungsstufen durch Versandapotheken, die Pharmahersteller und Patienten(-gruppen) direkt miteinander verbinden, wäre das klassische Beispiel einer Reintermediation (Wirtz 2001, S. 161) oder Transintermediation (Picot/Reichwald/Wigand 2003, S. 381 ff.) in der Internet-Ökonomie.

Die aktuelle Gesundheitsreform sieht nur eine Lockerung des Mehrbesitzverbotes vor. Ein selbständiger Apotheker kann auf dieser Grundlage insgesamt bis zu vier Apotheken betreiben. Weiterhin bleibt das Fremdbesitzverbot bestehen. Diese Veränderung bietet zunächst keine ausreichende Grundlage zur Bildung erfolgreicher Apothekenketten. Ob das Gesetz in der derzeitigen Form jedoch vor deutschen und europäischen Gerichten Bestand haben kann, wird von vielen Experten bezweifelt [10].

\subsection{Isolationsmechanismen zentraler Ressourcen und Kompetenzen sowie Ansätze zu ihrer Überwindung}

Wirken Isolationsmechanismen einerseits als Schutz nachhaltiger Wettbewerbsvorteile, so können sie andererseits im Falle geplanter Kooperationen auch als $\mathrm{zu}$ überwindendes Hindernis interpretiert werden (Gersch 2002, S. 426 ff.; Sanchez et al. 1996, S. 8 f.). Je nach Kompetenzprofil möglicher Kooperationspartner muss es gelingen, die jeweils vorhandenen Stärken der Akteure in ein konkretes Geschäftssystem einzubringen. Relevante Ressourcen und Kompetenzen sind aber z. B. in Form von implizitem Mitarbeiterwissen (z. B. Category Management oder One-to-One-Marketing-Know-how) oder komplexen Ressourcenbündeln (z. B. Bündel aus Prozessabläufen und IuK-Systemen im Fall von artikelgenauen unternehmensübergreifenden Computergestützten Warenwirtschaftssystemen oder CRM-Anwendungen) in den Potenzial- und Prozessstrukturen der jeweiligen Akteure gebunden (Gersch 2002, S. 426 f.). Diese Isolationsmechanismen zielgerichtet $\mathrm{zu}$ überwinden, stellt hohe Anforderungen an das Organisationsmodell des kooperativ zu realisierenden Geschäftssystems.

Neben den Aspekten der funktionellen Übertragung sind aber insbesondere auch die Vorbehalte potenzieller Kooperationspartner gegenüber einer drohenden Erosion und/oder Diffusion relevanter Ressourcen und Kompetenzen zu beachten. Auch in traditionellen Geschäftsfeldern werden erfolgskritische Bereiche nicht ohne ent- 
sprechende Absicherungen in denkbare Kooperationen eingebracht.

Die hier nur ansatzweise genannten Problembereiche bestimmen die Anforderungen der Beteiligten an die zu gestaltende Kooperationsform zur Realisierung des Geschäftssystems Versandapotheke. Bevor nicht die Bedürfnisse der Akteure berücksichtigende Organisationsdesigns der Kooperation gefunden werden, können die zur Überwindung der Isolationsmechanismen notwendigen Maßnahmen (z. B. Abstellen von Mitarbeitern mit entsprechendem Know-how für Aufbau und Betrieb benötigter Leistungserstellungspotenziale; Erweiterung und Anpassung vorhandener Kapazitäten im Rahmen eines notwendigen „Competence Leveraging“ bzw. „Competence Building“; siehe Gersch 2002, S. 424 ff.) nicht ergriffen werden.

\subsection{Grundsätzlich geeignet erscheinende Koope- rationen zur Realisierung des Geschäftssystems Versandapotheke}

Die konkrete Gestaltung denkbarer Kooperationen ist mit einer Vielzahl von Herausforderungen verbunden (u. a. rechtliche Ausgestaltung und Absicherung der $\mathrm{Zu}$ sammenarbeit, Lösung zu erwartender Kooperationskonflikte, Gestaltung der konkreten operativen Leistungserstellung etc.), die hier nur ansatzweise genannt werden können [12]. Auch können aus den skizzierten Szenarien nur zwei denkbare Kooperationen exemplarisch aufgegriffen und kurz skizziert werden. Aufgrund der augenfällig besten Ausgangsvoraussetzung scheint der Pharmagroßhandel eine entscheidende Rolle bei der Etablierung von Versandapotheken einzunehmen. Aus der Perspektive fehlender Kompetenzen bedarf es aber insbesondere der Ergänzung um Vermarktungs-Know-how im Endkonsumenten-Bereich und um Konzepte zur Überwindung der letzten Meile. Denkbar wäre daher die Kooperation mit einem Logistikdienstleister. Ohne die Einschaltung von Apothekern fehlen allerdings notwendige rechtliche Voraussetzungen. Darüber hinaus können die PHGH auf absehbare Zeit nicht ihre Hauptkunden - die derzeit noch 21.465 öffentlichen Apotheken - durch eindeutige Disintermediation verprellen. Wahrscheinlicher erscheinen kooperative Lösungen unter Einschaltung selektierter Apotheken, die sich als stationäre Outlets in ein „Mehrkanal-Konzept" einbringen können. So wären auch Defizite reiner Fernabsatzkonzepte, beispielsweise durch das optionale Angebot des persönlichen Gesprächs mit involvierten Apothekern, zu vermeiden.

Das zu realisierende Geschäftssystem Versandapotheke ist dauerhaft zu etablieren. Die durch Kooperationspartner einzubringenden Ressourcen und Kompetenzen zur Schaffung wettbewerbsfähiger Potenzial- und Prozessstrukturen erscheinen nicht durch Entwicklung eigener Kapazitäten in der erforderlichen Zeit, Qualität und Effizienz möglich (auch nicht unter Miteinbeziehung möglicher Dienstleister in einer Aufbauphase) [10]. Die Vorteile des Pharmagroßhandels hinsichtlich vorhandener
Ressourcen und Kompetenzen überwiegen derart, dass zwar auf Dauer angelegte Kooperationsformen wie Strategische Allianzen in Ausprägungen wie dem Joint Venture (Gersch 2002, S. 431 ff.) grundsätzlich möglich sind, realistischer erscheint aber die noch stärkere Anbindung der - relativ kleineren - Apotheken als Kooperationspartner durch Kapitalbeteiligungen oder beispielsweise durch Realisierung von Franchise-Konzepten. Hierzu wäre allerdings die Aufhebung des Fremd- und Mehrbesitzverbotes bei Apotheken (nach \$7 ApoG) zwingende Voraussetzung. Es wäre der Einstieg (oder die Ergänzung) von Apothekenketten unter der Leitung nationaler oder internationaler Pharmagroßhändler. Sollen Versandapotheken ohne diese Möglichkeiten, aber unter Beteiligung des PHGH realisiert werden, bleiben sie entweder auf den Bereich genossenschaftlich durch Apotheken getragener PHGH (wie z. B. die NOWEDA) beschränkt oder sie werden durch $z$. B. enge vertragliche Bindungen zwischen partizipierenden Apotheken und dem jeweiligen PHGH abgesichert. Ausländische PHGH verfügen derzeit nicht über die notwendige Infrastruktur in Deutschland, könnten aber versuchen, den Umweg der Übernahme eines deutschen PHGH zu gehen (Ossenberg-Engels 2002).

Eine Realisierung ohne die Beteiligung des PHGH müsste entweder auf kleine sachliche/regionale Nischen begrenzt bleiben oder würde den Nachbau der skizzierten logistischen PHGH-Infrastruktur erfordern. Dies und die notwendige Mindestgröße zur Erreichung wettbewerbsfähiger Einkaufskonditionen über das auch zukünftig gesetzlich vorgeschriebene Vollsortiment wäre prinzipiell erst ab einer Geschäftssystemgröße jenseits 250 Mio. Euro denkbar und auch dann kaum wahrscheinlich (Kerckhoff 2002; Ossenberg-Engels 2002).

\section{Ausblick}

Das Beispiel der Versandapotheke zeigt, dass E-Commerce-Geschäftssysteme regelmäßig deutliche Unterschiede zu traditionellen Branchen und Betätigungsfeldern aufweisen. Kein potenzieller Akteur allein verfügt über alle notwendigen Ressourcen und Kompetenzen, um ein Geschäftssystem mit den benötigten Potenzialund Prozessstrukturen zur wettbewerbsfähigen Leistungserstellung autonom zu realisieren. Kooperationen bieten sich zur Realisierung an. Scheinen einzelne Apotheken bzw. Apotheker - insbesondere auch aufgrund der auf absehbare Zeit gültigen Grundsätze der Reglementierung des Arzneimittelmarktes - zunächst unverzichtbar, so zeigt die Diskussion möglicher Väter als ergänzende Akteure zur Realisierung von Versandapotheken, dass insbesondere die derzeitigen deutschen Pharmagroßhändler hier die besten Ausgangspositionen haben.

Sollten zukünftige rechtliche Rahmenbedingungen durch Wegfall des aktuell noch gültigen Fremdbesitzverbotes die Bildung auch größerer Apothekenketten ermöglichen, wäre eine konsequente Multi-Channel-Strategie 
deutscher Großhändler, insbesondere auch zur Abwehr denkbarer Aktivitäten ausländischer Akteure und drohender Disintermediationstendenzen, wahrscheinlich. Der Gesetzgeber hat mit der Gesundheitsreform 2003/ 2004 einen ersten Schritt getan und den Beginn einer „eTransformation“ angestoßen.

Aufgrund der starken Position des PHGH wird sein zukünftiges Verhalten darüber bestimmen, ob es überhaupt zur Etablierung neuer Geschäftssysteme mit z. T. innovativen (E-)Service-Angeboten kommen wird [12]. Zentrale Aspekte zukünftiger Geschäftssysteme können zum jetzigen Informationsstand nur vermutet und auf der Basis theoretischer Überlegungen ansatzweise prognostiziert werden. Der sich verändernde Markt bleibt auch zukünftig ein hoch interessantes Betätigungsfeld einer anwendungsorientierten Betriebswirtschaftslehre mit einer Vielzahl relevanter Fragestellungen.

\section{Anmerkungen}

[1] Synonym auch „New Economy“. Gemeint ist hiermit das Handeln in einer Volkswirtschaft mit durch Digitalisierung und Vernetzung geprägten Rahmenbedingungen, die z. T. auch zu veränderten oder neuen Regeln für die Akteure führen (siehe hierzu auch Wirtz, 2001, S. 18 ff.).

[2] Hierbei kennzeichnet „Coompete“ oder „Coopetition“ das in der Internet-Ökonomie vermehrt zu beobachtende Phänomen der beiderseitigen Vorteilhaftigkeit bei der Kooperation von potenziellen und/oder aktuellen Konkurrenten (Bengtsson/ Kock 2000, S. 414 ff.; Gersch 2000b, S. 2 f.).

[3] Mit dem Begriff Geschäftsmodell wird hier die Abbildung des betrieblichen Produktions- und Leistungssystems eines Unternehmens oder einer Kooperations-/Anbietergemeinschaft bezeichnet. In stark vereinfachter und aggregierter Form wird dargestellt, welche Ressourcen in die Unternehmung fließen und wie diese durch innerbetriebliche und kooperative Erstellungsprozesse in vermarktungsfähige Leistungsbündel für als relevant erachtete Märkte transformiert werden. Ansatzpunkte zur Generierung von Erlösen und Kosten durch eine Geschäftstätigkeit werden aufgezeigt und somit die Grundlagen für Erfolg oder Misserfolg analysiert (siehe hierzu auch Wirtz 2001, S. 210 ff.). Der Begriff Geschäftssystem kennzeichnet die konkrete Realisierung eines allgemeinen Geschäftsmodells. Die niederländische InternetApotheke „Doc Morris“ ist nach diesem Begriffsverständnis ein konkretes Geschäftssystem des allgemeinen Geschäftsmodells „Merchant“ nach Rappa (2002) bzw. „E-Shop“ nach Timmers (1998).

[4] Im Rahmen des vorliegenden Beitrages werden die Begriffe Versand- und Internetapotheke verwendet. Versandapotheke ist dabei der umfassendere Begriff eines Geschäftssystems im Bereich Fernabsatz mit Medikamenten auf der Basis der Empfehlung des runden Tisches im Bundesministerium für Gesundheit vom April 2002 (Vollsortiment; Nacht- und Notdienst; Lieferzwang; Leitung durch einen Apotheker) und der Gesundheitsreform 2003/2004 [5]. Es handelt sich somit um eine öffentliche Apotheke mit Sondervertriebsweg durch Aufhebung des §43,I Arzneimittelgesetz sowie §17, I Apothekenbetriebsordnung. Internetapotheke kennzeichnet in diesem Sinne nur einen speziellen Kommunikations- und Bestellkanal zum Endkunden. Erfolgt die verbindliche Bestellung der Leistungen via Internet, so handelt es sich bei diesem Typus der Versandapotheke um ein E-Commerce-Geschäftssystem (Gersch 2000a, S. 3 ff.; Sterzel/Wassener 2001, S. 17).
[5] Die aktuelle Gesundheitsreform 2003/2004 wird als GMG (,Gesetz zur Modernisierung der Gesetzlichen Krankenversicherung") ab dem 1. Januar 2004 in Kraft treten. Wichtige Aspekte sind die Aufhebung des Versandhandelsverbotes, die Lockerung des Mehrbesitzverbotes unter Beibehaltung des Fremdbesitzverbotes (ein unabhängiger Apotheker kann zukünftig insgesamt maximal 4 Apotheken betreiben) sowie eine Veränderung der Preisregulierung (insb. Möglichkeiten zur Schließung von Einzelverträgen zwischen Kostenträgern und Leistungserbringern sowie die teilweise Einführung einer Einzelentgeltung von Apothekenleistungen) (BMG 2003).

[6] Siehe hier und im Folgenden Wirtz (2001, S. 210 ff.). Die Teilmodelle von Wirtz wurden modifiziert und insbesondere um das Organisationsmodell ergänzt. Das Organisationsmodell wird in Abschnitt 3 diskutiert.

[7] Die in $A b b .2$ skizzierte Struktur trägt 2001 folgende Größenordnungen: 70 Mio. Versicherte, 360 Krankenkassen, 110.000 Ärzte, 53.000 Zahnärzte, 1.100 Pharmahersteller, 21.569 öffentliche Apotheken, 2.200 Krankenhäuser mit 563 Krankenhausapotheken sowie 120.000 sonstige Leistungserbringer erzeugen jährlich ca. 600 Mio. Rezepte mit 900 Mio. Verordnungen. Apothekenpflichtige Arzneimittel generieren hierbei 27 Mrd. Euro Umsatz (ohne MwSt.) auf Seiten der Apotheken, von denen 6,7 Mrd. nicht verschreibungspflichtig waren (ABDA 2003; Pfaff et al. 2002, S. 62).

[8] Disease Management Konzepte kennzeichnen strukturierte Behandlungsprogramme, die sich speziell auf bestimmte Patientengruppen (z. B. chronisch Kranke oder Transplantationspatienten) konzentrieren und so eine koordinierte und qualitativ hochwertige Versorgung über verschiedene Fachbereiche der Medizin hinweg aus einer Hand organisieren. Seit dem 1. Juli 2002 hat der deutsche Gesetzgeber derartige Service-Angebote, die als Intermediäre etablierte Versorgungsstrukturen ergänzen, nach $§ 137 f$ SGB V zugelassen (Bundesärztekammer 2002).

[9] In Anlehnung an Bruhn (2002, S. 6) werden so genannte Electronic Services (synonym auch E-Services) wie folgt definiert: Electronic Services sind selbständige, marktfähige Leistungen, die durch die Bereitstellung von elektronischen Leistungsfähigkeiten des Anbieters (Potenzialdimension) und durch die Integration externer Faktoren (u. a. mit Hilfe des elektronischen Datenaustausches) (Prozessdimension) auf eine nutzenstiftende Wirkung (u. a. durch Be- oder Verarbeitung externer Faktoren) (Ergebnisdimension) abzielen.

Charakteristisches Merkmal ist die Nutzung der Informationstechnologie bei der Gestaltung der Potenzial-, Prozessund Ergebnisdimension integrativ erstellter Leistungsangebote. Für den Kunden insbesondere wahrnehmbar sind die mit E-Services zumeist verbundenen Mensch-Maschine-Interaktionen an wichtigen Kundenkontaktpunkten.

[10] Dies ist das Ergebnis mehrerer Expertendiskussionen, die am Competence Center E-Commerce der Ruhr-Universität Bochum seit August 2002 geführt wurden. Hierzu gehören auch Kerckhoff (2002); Ossenberg-Engels (2002); Zocholl (2003). Einzelne Ergebnisse sind ausführlicher dokumentiert in Gersch/Theißen (2003).

[11] Hierbei ist zu beachten, dass auch nach der Gesundheitsreform 2003/2004 selbständige Apotheker verantwortliche Träger des Geschäftssystem Versandapotheke sein müssen (BMG 2003). Es ist in näherer Zukunft nicht mit einer gänzlichen Aufhebung des Fremdbesitzverbotes zu rechnen. Hierdurch entfallen zunächst die Optionen einzelner Akteure zur autarken Realisierung eines Geschäftssystems. Die als Überblick zusammen gefassten Informationen basieren auf sehr ausführlichen Ergebnissen einer Reihe von Expertendiskussionen [10].

[12] Die zukünftige Entwicklung ist darüber hinaus wesentlich von (z. T. politischen) Entscheidungen unterschiedlicher Ebe- 
nen (insb. Europäischer Gerichtshof; europäische und deutsche Legislative) abhängig. Diese werden jeweils die relevanten Rahmenbedingungen für die diskutierten Akteure bilden. Das exakte Ergebnis des zu erwartenden Diffusionsprozesses neuer Dienstleistungsangebote im Rahmen innovativer Geschäftssysteme kann nicht prognostiziert werden. Zur Begleitung dieses dynamischen Entwicklungsprozesses wurde am Competence Center E-Commerce der Ruhr-Universität Bochum eine multimediale Online-Fallstudie entwickelt, die die Diskussion der jeweils aktuellen Entwicklungen ermöglicht.

\section{Literaturverzeichnis}

ABDA (2003): Apotheken: Zahlen, Daten, Fakten 2002, http://www.abda.de/ABDA/datenrechner.html, abgerufen am 16. Oktober 2003.

Ahlert, D. (2000): Implikationen des Electronic Commerce für die Akteure in der Wertschöpfungskette, in: Ahlert, D./Becker, J./ Kenning, P./Schütte, R. (Hrsg.): Internet \& Co. im Handel, Berlin u. a., S. 3-27.

Backhaus, K. (2003): Industriegütermarketing, 7. Aufl., München.

Backhaus, K./Meyer, M. (1993): Strategische Allianzen und Strategische Netzwerke, in: WiSt, 22. Jg., Nr. 7, S. 330-334.

Backhaus, K./Piltz, K. (1990): Strategische Allianzen - eine neue Form kooperativen Wettbewerbs?, in: Backhaus, K./Piltz, K. (Hrsg.): Strategische Allianzen, Sonderheft 27 der Zeitschrift für betriebswirtschaftliche Forschung, S. 1-10.

Badenhoop, R./Seiter, S./Emrich, K. (2002): Versandhandel Arzneimittel in den USA - ein Modell für Deutschland?, Internationale Studie der Gesellschaft Cap Gemini Ernst \& Young, Berlin, http://www.cgey.com/servlet/PB/menu/1004849/undex.html, abgerufen am 2. Oktober 2002.

Baden-Fuller, C.Nolberda, H.W. (1997): Strategic renewal in large complex organizations: A competence-based view, in: Heene, A./Sanchez, R. (Eds.): Competence-based strategic management, Chichester, pp. 89-110

Bamberger, I./Wrona, T. (1996a): Der Ressourcenansatz und seine Bedeutung für die Strategische Unternehmensführung, in: Zeitschrift für betriebswirtschaftliche Forschung, 48. Jg., Nr. 2 , S. 130-153.

Bamberger, I./Wrona, T. (1996b): Der Ressourcenansatz im Rahmen des Strategischen Managements, in: Wirtschaftswissenschaftliches Studium, 25. Jg., Nr. 8, S. 386-391.

Barney, J.B. (1991): Firm Resources and Sustained Competitive Advantage, in: Journal of Management, Vol. 17, No. 1, pp. 99120.

Becker, J./Uhr, W./Vering, O. (2000): Integrierte Informationssysteme im Handelsunternehmen auf der Basis von SAP-Systemen, Berlin u. a.

Bengtsson, M./Kock, S. (2000): „Coopetition“ in Business Networks - to cooperate and compete simultaneously, in: Industrial Marketing Management, Vol. 29, pp. 411-426.

Bruhn, M. (2002): E-Services - eine Einführung in die theoretischen und praktischen Probleme, in: Bruhn, M./Stauss, B. (Hrsg.): Electronic Services. Dienstleistungsmanagement Jahrbuch 2002, Wiesbaden, S. 3-42.

Bundesärztekammer (2002): http://www.bundesaerztekammer.de/ 30/Qualitaetssicherung/58DMP, abgerufen am 12. Dezember 2002.

Bundesministerium für Gesundheit BMG (2002a): Argumente zu Versandhandel von Arzneimitteln, http://www.bmgesundheit.de/ bmg-frames/index.htm, abgerufen am 25.08.2002.

Bundesministerium für Gesundheit BMG (2002b): Empfehlungen des ,Runden Tisches' auf seiner 4. Sitzung am 22. April 2002 zum elektronischen Handel einschließlich Versandhandel mit Arzneimitteln, http://www.bmgesundheit.de/downloads-themen/rundertisch/ elektrhandel.doc, abgerufen am 25.08.2002.

Bundesministerium für Gesundheit BMG (2003): Die Gesundheitsreform 2003/2004, http://www.die-gesundheitsreform.de, abgerufen am 21.11.2003.
Däinghaus, $R$. (2003): Internet-Versandhandel und die Strukturen des Arzneimittelmarktes, in: Oberender, P. (Hrsg.): Wettbewerb in der Internetökonomie, Berlin, S. 97-114.

Dierickx, I./Cool, K. (1989): Asset Stock Accumulation and Sustainability of Competitive Advantage, in: Management Science, Vol. 35, No. 12, pp. 1504-1511.

Engelhardt, W.H./Gersch, M. (1995): Informationsmanagement als Instrument zur erfolgreichen Gestaltung von Geschäftsbeziehungen - am Beispiel des deutschen Pharmagroßhandels, in: Trommsdorf, V. (Hrsg.): Handelsforschung 1995/96 - Informationsmanagement im Handel, Jahrbuch der Forschungsstelle für den Handel (FfH), Berlin, S. 201-222.

Engelhardt, W.H./Reckenfelderbäumer, M. (1993): Trägerschaft und organisatorische Gestaltung industrieller Dienstleistungen, in: Simon, H. (Hrsg.): Industrielle Dienstleistungen, Stuttgart, S. $263-293$.

Freiling, J. (1998a): Kompetenzorientierte Strategische Allianzen, in: io Management, 67. Jg., Nr. 6, S. 23-29.

Freiling, J. (1998b): Rüstzeug für den Ressourcen Check-up. Kompetenz-Analyse Teil I, in: Absatzwirtschaft, 41. Jg., Nr. 4, S. 70-76.

Freiling, J. (1998c): Rüstzeug für den Ressourcen Check-up. Kompetenz-Analyse Teil II, in: Absatzwirtschaft, 41. Jg., Nr. 5 , S. 74-77.

Freiling, J. (2001): Ressourcenorientierte Reorganisation, Wiesbaden.

Freiling, J. (2002): Terminologische Grundlagen des Resourcebased View, in: Bellmann, K. et al. (Hrsg.): Aktionsfelder des Kompetenz-Management, Wiesbaden, S. 3-28.

Frey, E. (1999): „Indikation Versandapotheke“ - Risiken und Nebenwirkungen des Arzneimittel-Versandhandels, in: Mattmüller, R. (Hrsg.): Versandhandelsmarketing, Frankfurt a. M., S. $553-578$.

Gahl, A. (1990): Die Konzeption Strategischer Allianzen im Spannungsfeld zwischen Flexibilität und Funktionalität, in: Backhaus, K./Piltz, K. (Hrsg.): Strategische Allianzen, Sonderheft 27 der Zeitschrift für betriebswirtschaftliche Forschung, S. 35-48.

Gahl, A. (1991): Die Konzeption Strategischer Allianzen, Berlin.

Gersch, M. (1995): Die Standardisierung integrativ erstellter Leistungen, Arbeitsbericht Nr. 57 des Instituts für Unternehmungsführung und Unternehmensforschung, Bochum.

Gersch, M. (1998): Vernetzte Geschäftsbeziehungen, Wiesbaden.

Gersch, M. (1999): Das Management vernetzter Geschäftsbeziehungen, in: Hippner, H./Meyer, M./Wilde, K. (Hrsg.): Computer Based Marketing, 2. Aufl., Braunschweig/Wiesbaden, S. 25-34.

Gersch, M. (2000a): E-Commerce - Einsatzmöglichkeiten und Nutzungspotenziale, Arbeitsbericht CCEC 1 und Nr. 82 des IUU, Bochum.

Gersch, M. (2000b): Die Herausforderung E-Commerce, in: Engelhardt, W.H./Gabriel, R./Gersch, M. (Hrsg.): Gründungsworkshop CCEC, Arbeitsbericht CCEC 2 und Nr. 84 des IUU, Bochum, S. 1-12.

Gersch. M. (2001): E-Commerce. Alte Hasen, Boomer und Schläfer, in: Verein Deutscher Ingenieure VDI (Hrsg.): Ingenieur Forum Westfalen-Ruhr, 3/2001, S. 4-6.

Gersch, M. (2002): Kooperationen als Instrument des KompetenzManagement zur Realisierung erfolgreicher E-Commerce-Geschäftssysteme, in: Bellmann, K./Freiling, J./Hammann, P./Mildenberger, U. (Hrsg.): Aktionsfelder des Kompetenz-Managements, Wiesbaden, S. 411-438.

Gersch, M. (2003): Cooperation as Instrument of Competence Management, in: International Journal of Management and Decision Making (IJMDM), Vol. 4, Nos. 2/3, pp. 210-229.

Gersch, M./Theißen, J. (2003): Konzeption und Analyse eines internet-gestützten E-Commerce-Geschäftssystems für Medikamente, Arbeitsbericht Nr. 9 des CCEC und Nr. 96 des IUU, Bochum.

Gersdorff, A. von (2002): Der Kampf der Apotheker, in: Die Welt vom 25. Juli 2002, S. 16 
Hammann, P./Freiling, J. (Hrsg.) (2000): Die Ressourcen- und Kompetenzperspektive des Strategischen Managements, Wiesbaden.

Hofmann, S./Fröndhoff, B. (2002): Pharmahändler haben Appetit, in: Handelsblatt, Nr. 182 vom 21. September 2002, S. 17.

Kerckhoff, T. (2002): Versandapotheken und Managed Care, Vortrag und Expertendiskussion am Competence Center E-Commerce der Ruhr-Universität Bochum, 4. Dezember 2002.

Kunz, A. (2001): Alternative Distributionswege für pharmazeutische Produkte, Wiesbaden.

Metzenthin, R. (2000): Kompetenzorientierte Mergers \& Acquisitions, in: Hammann, P./Freiling, J. (Hrsg.): Die Ressourcenund Kompetenzperspektive des Strategischen Managements, Wiesbaden, S. 277-310.

Meyer, M. (1995): Ökonomische Organisation der Industrie, Wiesbaden.

Mihm, A. (2002): Das Gespenst der Apotheker, in: Frankfurter Allgemeiner Zeitung, Nr. 195 vom 23. August 2002, S. 11.

Ossenberg-Engels, J. (2002): Internetapotheken und die Zukunft des Deutschen Gesundheitswesens, Vortrag und Expertendiskussion am Competence Center E-Commerce der Ruhr-Universität Bochum, 12. Dezember 2002.

Picot, A./Reichwald, R./Wigand, R. (2003): Die grenzenlose Unternehmung, 5. Aufl., Wiesbaden.

Pfaff, M./Wassener, D./Sterzel, A./Neldner, T. (2002): Analyse potentieller Auswirkungen einer Ausweitung des Pharmaversandes in Deutschland, Allokation im marktwirtschaftlichen System, Band 45, Frankfurt a. M. u. a.

Prinz, A./Vogel, A. (2002): Electronic Commerce im Arzneimittelhandel: Zulassen, verbieten oder regulieren? Gutachten für den Deutschen Bundestag, Münster.

Rappa, M. (2002): Managing the Digital Enterprise: Business Models, North Carolina State University, http://ecommerce.ncsu.edu/ topics/models/models.html, abgerufen am 16. Oktober 2002.

Reiß, M. (2001): Netzwerk-Kompetenz, in: Corsten, H. (Hrsg.): Unternehmensnetzwerke - Formen unternehmensübergreifender Zusammenarbeit, München, S. 121-188.

Riehm, U./Petermann, T./Orwat, C. et al. (2002): E-Commerce Endbericht, Arbeitsbericht Nr. 78 des Büros für Technikfolgeabschätzung beim Deutschen Bundestag, Juni 2002.

Ruess, A. (2002): Geschütztes Monopol, in: Wirtschaftswoche, Nr. 36 vom 29. August 2002, S. 48-49.

Sanchez, R./Heene, A./Thomas, H. (1996): Towards the theory and practice of competence-based competition, in: Sanchez, R./ Heene, A./Thomas, H. (Eds.): Dynamics of Competence-based Competition: Theory and Practice in the new strategic management, Oxford, pp. 1-35.

Schmid, B. (2001): What is new about the Digital Economy?, in: Electronic Markets, Vol. 11, No. 1, pp. 44-51.

Schneider, D. (1998): Vorläufer der Lehre von den Kernkompetenzen: von Xenophons Kyrupaedie über Babbage's Prinzip zu Lists „Gesetz der Kraftvereinigung“, in: Glaser, H./Schröder, E./Werder, A. von (Hrsg.): Organisation im Wandel der Märkte, Wiesbaden, S. 343-358.

Shapiro, C./Varian, H. (1998): Information Rules: A Strategic Guide to the Network Economy, Boston.

Sterzel, A./Wassener, D. (2001): Arzneimitteldistribution in Deutschland - Versandhandel als Reformoption?, in: Arbeit und Sozialpolitik, 55. Jg., Nr. 1-2, S. 14-20.

Timmers, P. (1998): Business Models for Electronic Markets, in: Electronic Markets, Vol. 8, No. 2, pp. 3-8.

Weiber, R. (Hrsg.) (2002): Handbuch Electronic Business, 2. Aufl., Wiesbaden.

Wirtz, B. (2001): Electronic Business, 2. Aufl., Wiesbaden.

Zerdick, A./Picot, A./Schrape, K. et al. (Hrsg.) (2001): Die InternetÖkonomie: Strategien für die digitale Wirtschaft, 3. Aufl., Berlin.
Zocholl, D. (2003): Defizite des traditionellen Gesundheitswesens - Neue Chancen für innovative Dienstleister, Vortrag und Expertendiskussion am Competence Center E-Commerce der Ruhr-Universität Bochum, 20. Juni 2003.

\section{Summary}

It is an interesting detail of the present reform of national public health service in Germany (GMG 2004) that mail-order- and internet pharmacies will be founded as (e-commerce) business models. Main points of conceivable business models can be characterized as well as critical success factors. Unlike established protagonists mail-order pharmacies will offer innovative (e-)services and they will disor reintermediate traditional industry structures. Because of current bills it seems that a pharmacist is also in the future a necessary part of a business model. But who will act as suitable 'father'?

No potential participant can ever have all necessary resources and competences to implement a competitive business model without external support. This is why cooperation seems to be a useful tool for competence management. The resource-based view will serve as theoretical background for the analysis. The required and available competences of conceivable players can be identified. Features of resources can be described that recommend special types of cooperations with the intention to establish and operate a business model. Especially German and international pharmaceutical wholesalers can be identified as conceivable 'fathers' of mail-order pharmacies in the future.

\section{Schlüsselbegriffe}

Versandapotheke, Internet-Apotheke, E-Commerce-Geschäftssystem, Resource-based View, Dis-/Reintermediation, (Electronic) Services

\section{Keywords}

Mail-order pharmacies, Internet pharmacies, e-commerce business model, resourcebased view, dis-/reintermediation, (electronic) services 


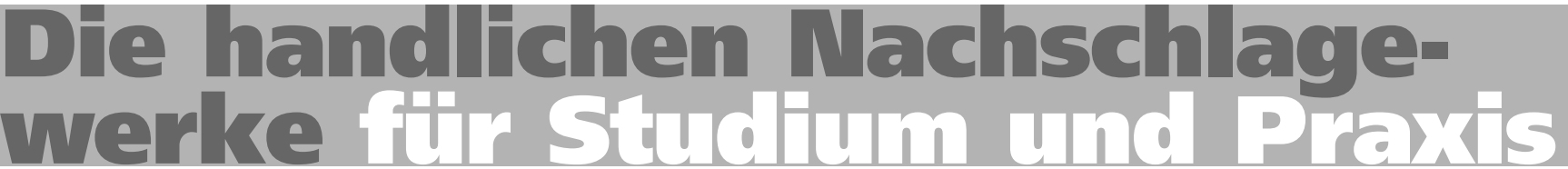

Das Financial Dictionary und das Management \& Marketing Dictionary sind bewährte Nachschlagewerke für Studium, Ausbildung und Praxis. Neben der laufenden Aktualisierung und Erweiterung des Wortbestandes setzen die vorliegenden Neuauflagen die Grundkonzeption des "handlichen Begleiters in allen Lebenslagen" noch konsequenter um: Die bisher auf jeweils zwei Bände verteilten Wörterbücher sind nun in je einem Band zusammengefasst. Unverändert bleibt die bewährte Ausrichtung an den Anforderungen der Praxis.

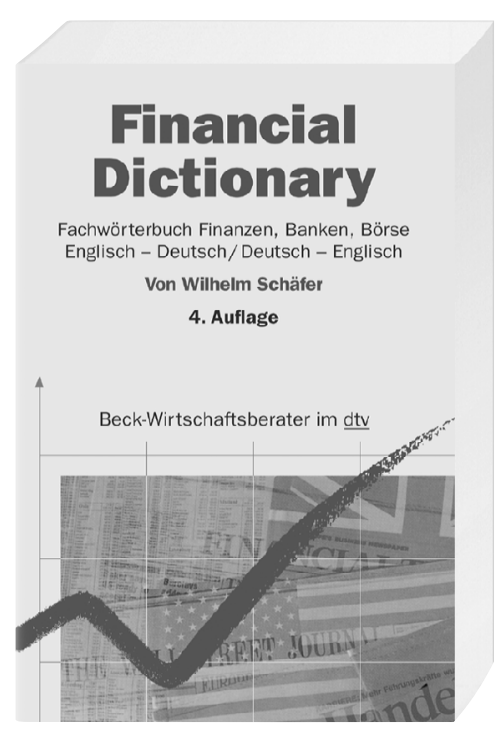

\section{Schäfer}

\section{Financial Dictionary}

Fachwörterbuch Finanzen, Banken, Börse Englisch-Deutsch/Deutsch-Englisch Begründet von Prof. Dr. Wilhelm Schäfer† Herausgegeben von Dr. Michael Schäfer

4. Auflage. 2004.

VIII, 887 Seiten. Kartoniert $€ 22$,-

(dtv-Band 50886)

Die Neuauflage ist vollständig überarbeitet und enthält inzwischen 30000 Stichwörter.

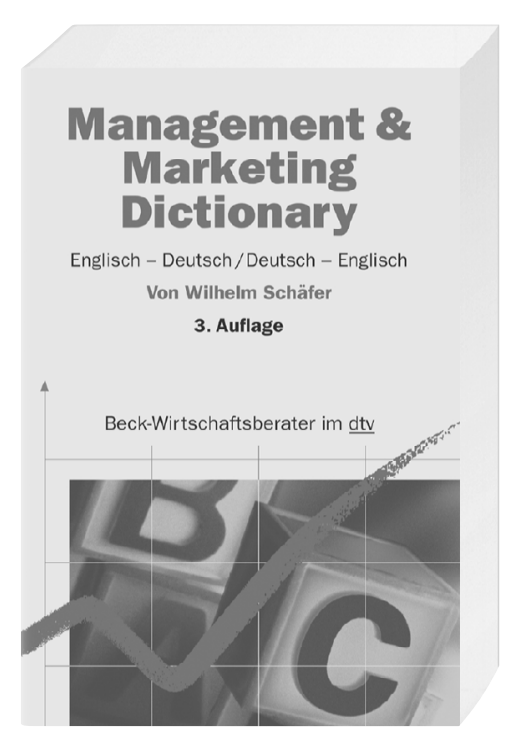

\section{Schäfer}

Management \& Marketing Dictionary

Englisch-Deutsch/Deutsch-Englisch Begründet von Prof. Dr. Wilhelm Schäfert Herausgegeben von Dr. Michael Schäfer 3. Auflage. 2004.

VIII, 760 Seiten. Kartoniert $€ 19,50$ (dtv-Band 50887)

Die Neuauflage ist vollständig überarbeitet und enthält mehr als 26000 Stichwörter.

\section{Verlag C.H. Beck im dtv}




\title{
Universelles Wissen
}

\section{Konzentrierte Kompetenz}

\author{
Das Wissen von mehr als $\mathbf{2 5 0}$ renommierten Autoren \\ aus Wissenschaft und Managementpraxis aus erster Hand.
}

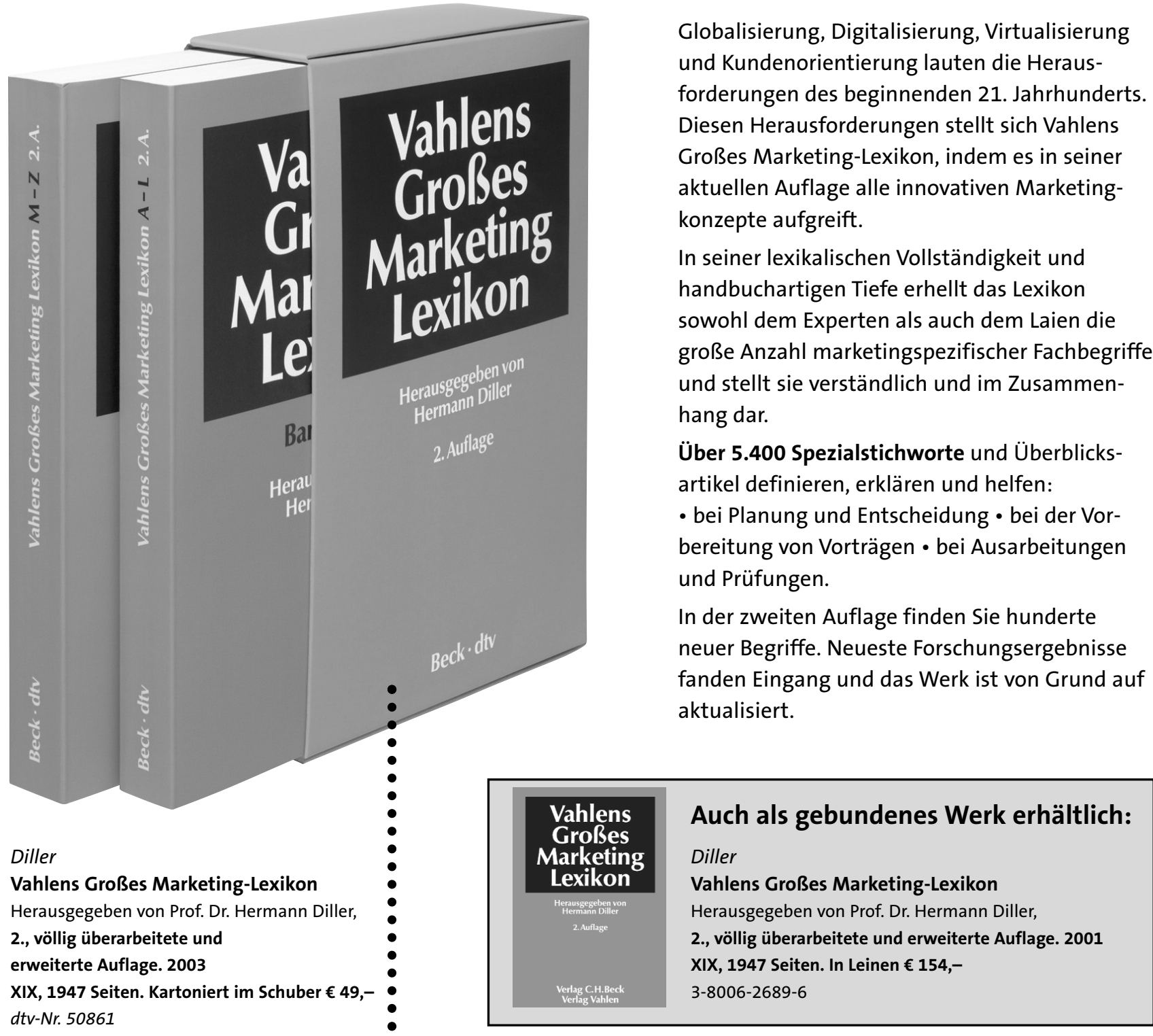

\section{FAX-COUPON}

Bitte bestellen Sie bei Ihrem Buchhändler oder bei:

Expl. dtv-Band 50861 Vahlens Großes Marketing-Lexikon

2. Auflage. 2003. Kartoniert im Schuber $€ 49$,- inkl. MwSt., zzgl. Vertriebskosten

Expl. 3-8006-2689-6 Vahlens Großes Marketing-Lexikon

2. Auflage. 2001. In Leinen $€ 154$,- inkl. MwSt., zzgl. Vertriebskosten

Sie haben das Recht, die Ware innerhalb von 2 Wochen nach Lieferung ohne Begründung an Ihre Buchhandlung oder an den Verlag Franz Vahlen, c/o Nördlinger Verlagsauslieferung, Augsburger Str. 67 a, 86720 Nördlingen zurückzusenden, wobei die rechtzeitige Augsburger Str. 67 a, 86720 Nördlingen zurückzusenden, wobel die
Absendung genügt. Kosten und Gefahr der Rücksendung trägt der Empfänger. Absendung genügt. Kosten und Gefahr der Rücksendung trägt
Ihr Verlag Franz Vahlen GmbH, Wilhelmstr. 9, 80801 München, Geschäftsführer: Dr. Hans Dieter Beck
Globalisierung, Digitalisierung, Virtualisierung forderungen des beginnenden 21. Jahrhunderts. Diesen Herausforderungen stellt sich Vahlens Großes Marketing-Lexikon, indem es in seiner aktuellen Auflage alle innovativen Marketingkonzepte aufgreift.

handbuchartigen Tiefe erhellt das Lexikon sowohl dem Experten als auch dem Laien die 作 hang dar. artikel definieren, erklären und helfen bereitung von Vorträgen • bei Ausarbeitungen und Prüfungen. neuer Begriffe. Neueste Forschungsergebnisse fanden Eingang und das Werk ist von Grund auf aktualisiert.

\section{Auch als gebundenes Werk erhältlich:}

Herausgegeben von Prof. Dr. Hermann Diller,

2., völlig überarbeitete und erweiterte Auflage. 2001

3-8006-2689-6 


\title{
Strategien und Trends
}

\section{im Handelsmanagement}

\author{
Disziplinenübergreifende \\ Herausforderungen und Lösungsansätze \\ Festschrift für Prof. Dr. Dr. h.c. Erich Greipl \\ Herausgegeben von \\ Prof. Dr. Hans H. Bauer und \\ Dipl.-Kfm. Frank Huber, Mannheim \\ 2004. X, 448 Seiten. \\ Gebunden $€$ 64,- \\ ISBN 3-8006-3104-0 \\ Erscheint im Juli 2004
}

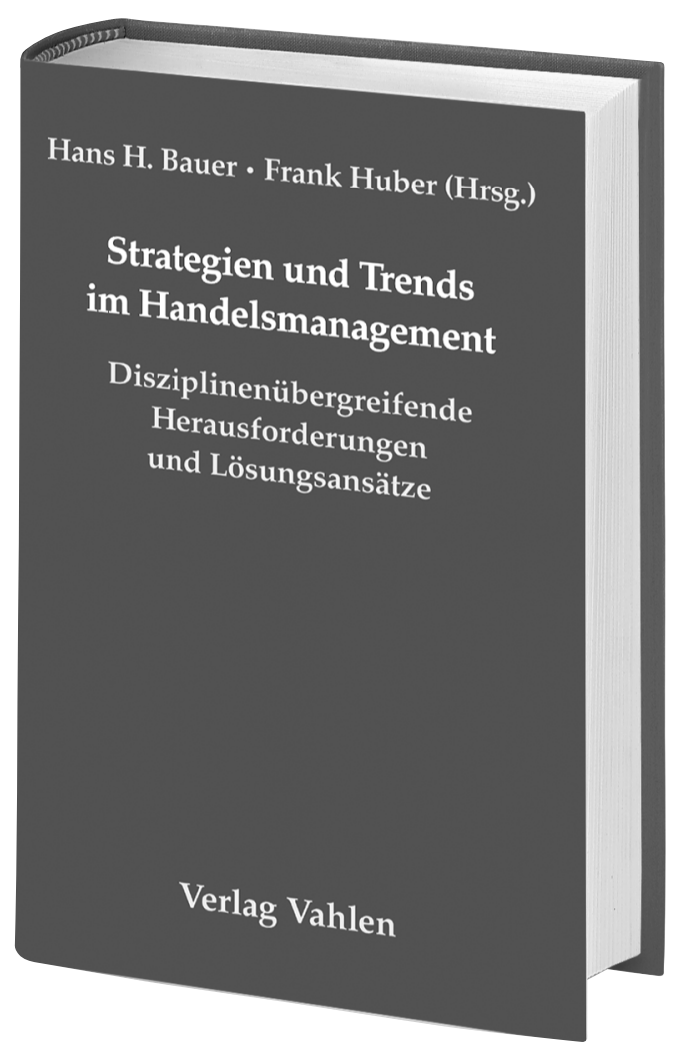

Ein sich veränderndes Konsumentenverhalten, Preisschlachten, Strukturveränderungen, Internationalisierungsbemühungen, neue Technologien sowie eine permanente Diskussion über staatliche Regulierungen und gesellschaftspolitische Einflüsse prägen sowohl strategische Überlegungen als auch das operative Tagesgeschäft deutscher Handelsunternehmen.
Die Festschrift zu Ehren des Handelsmanagers und Handelsforschers Prof. Dr. Dr. h.c. Erich Greipl enthält Beiträge, die aktuelle Herausforderungen des Handels thematisieren und Lösungsansätze anbieten. Hierbei werden sowohl spezifische Aspekte des klassischen Groß- und Einzelhandels diskutiert, als auch verwandte Bereiche, wie der Online-Handel oder für den Handel übertragbare Lösungsansätze anderer Branchen, vorgestellt.

\section{FAX-COUPON}

Expl. 3-8006-3104-0

Bauer/Huber, Strategien und Trends im Handelsmanagement 2004. Gebunden $€ 64$,- inkl. MwSt., zzgl. Vertriebskosten

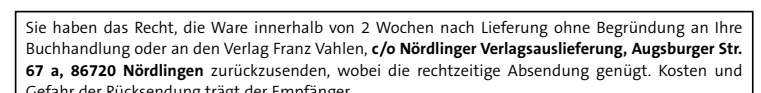
Buchhandlung oder an den Verlag Franz Vahlen, c/o Nördlinger Verlagsauslieferung, Augsburger Str. 67 a, 86720 Nördlingen zurückzusenden, wobei die rechtzeitige Absendung genügt. Kosten und

Gefahr der Rücksendung trägt der Empfänger.
Ihr Verlag Franz Vahlen GmbH, Wilhelmstr. 9, 80801 München, Geschäftsführer: Dr. Hans Dieter Beck

\begin{tabular}{ll} 
Name/Firma & $\begin{array}{l}\text { Bitte bestellen Sie } \\
\text { bei Ihrem Buchhändler } \\
\text { oder bei: }\end{array}$ \\
\hline Straße & \begin{tabular}{l} 
F0791 MÜNCHEN \\
Internet: www.vahlen.de \\
\hline PLZ/Ort
\end{tabular} \\
E-Mail:bestellung@vahlen.de \\
\end{tabular}




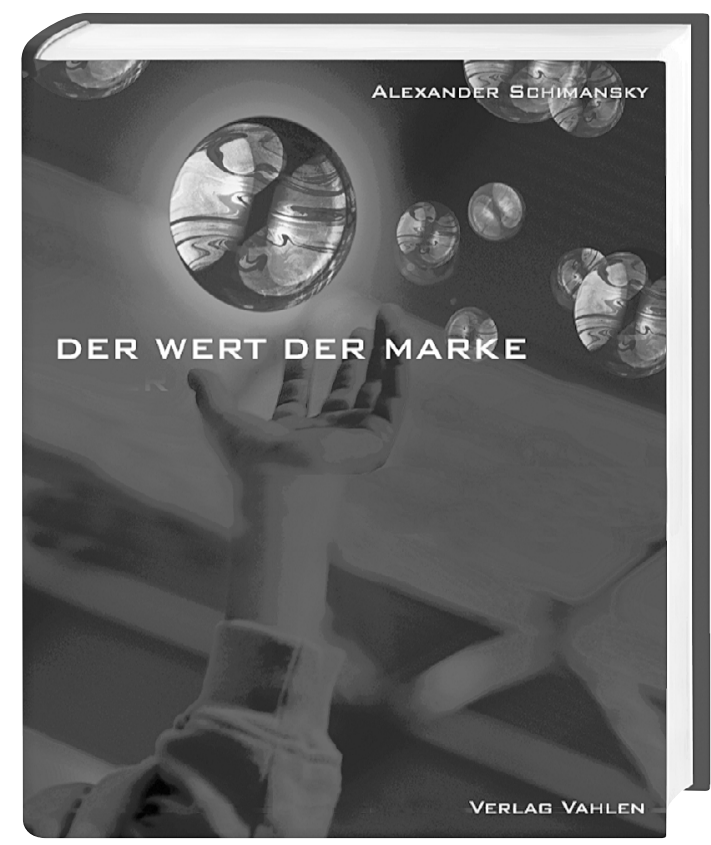

Erfolgreiche Markenführung ist mehr denn je zum Überlebensfaktor für Unternehmen geworden. Eine wichtige Voraussetzung für gewinnbringende Markenstrategien bildet das fundierte Verständnis von der eigenen Marke. Dieses Buch gibt erstmals einen detaillierten Überblick über relevante Ansätze zur Markenanalyse und Markenbewertung. Alle namhaften Verfahren im deutschsprachigen Raum, die in den letzten 15 Jahren entwickelt worden sind, werden ausführlich vorgestellt. Ziel ist es, sowohl Charakteristika als auch Gemeinsamkeiten und Unterschiede von Markenbewertungsansätzen darzustellen, um einen Eindruck über die Qualität und Möglichkeiten der zum Teil sehr verschiedenen Verfahren zu vermitteln.

Zudem wird über Ergebnisse aus einer eigenen empirischen Studie zur Bedeutung von Markenbewertungsansätzen in der heutigen Marketingpraxis berichtet. Ein Einblick in die bewegte Vergangenheit der Markenführung von Prof. Geldmacher und ein Ausblick auf die Zukunft der Markenwert-Entwicklung von Prof. Herbst runden das Werk ab.

Dieses hochwertig aufgemachte Buch ist ein Muss für Markendenker und -lenker. Besonders Entscheider in Profit- aber auch in Nonprofit-Unternehmen, die nach neuen Lösungswegen für aktuelle Markenprobleme suchen, werden von diesem Buch profitieren.

\section{Die Markenbewertungsansätze und deren Anbieter in alphabetischer Reihenfolge der Verfahren:}

- 360 Degree Brand Stewardship von Ogilvy \& Mather

- Advanced Brand Valuation von

PricewaterhouseCoopers

- Brand Ambassador von Adjouri: Brand Consultants

- Brand Assessment System von der GfK

- Brand Asset Valuator von Young \& Rubicam

- Brand Building Tools von Grey

- Brand Equity Evaluator von BBDO

- Brand Performance System von AC Nielsen / Konzept \& Markt

- BrandStock von Michael Conrad \& Leo Burnett

- Brand Value Creation von The Boston Consulting Group

- Brand Wheel von Bates Germany

- BrandZ von WPP / J. Walter Thompson

- Conversion Model von TNS Emnid

- CAPO von \&equity

- Der Genetische Code der Marke vom Institut für Markentechnik

- GIM-Values von der Gesellschaft für Innovative Marktforschung

- icon Brand Navigator von icon brand navigation

- Implizites System (Imp/Sys) von NFO Infratest

- Interbrand-Modell von Interbrand, Zintzmeyer \& Lux

- Ipsos*Builder von Ipsos Deutschland

- MarkenMatik von McKinsey

- MarkenMonopol-Ansatz von Konzept \& Analyse

- Markenwertbarometer von facit MarketingForschung

- MarkenWesen-Modell von Publicis-Sasserath
- Market Router von \Sturm und Drang (TBWA)

- Morphologische Markenkernanalyse von rheingold

- PromiCheck-Ansatz von Promikativ

- res+-Modell von we do communications

- Roadmap to Effective Communication von McCann-Erickson

- rb Profiler von Roland Berger Strategy Consultants

- semion brand valuation-Ansatz von semion brand-broker

- WISA von Prof. Dr. Volker Trommsdorff

\section{FAX-COUPON}

Expl. 3-8006-2984-4

Der Wert der Marke

2004. Gebunden $€ 98$,- inkl. MwSt., zzgl. Vertriebskosten

Name/Firma

Straße

$\overline{\text { PLZ/Ort }}$

\begin{tabular}{ll}
\hline Datum/Unterschrift & $\mathrm{B} / 134619$
\end{tabular}

Sie haben das Recht, die Ware innerhalb von 2 Wochen nach Lieferung ohne Begründung an Ihre Buchhandlung oder an den Verlag Franz Vahlen, c/o Nordlinger Verlagsauslieferung, Augsburger Str. $67 \mathrm{a}$ a, 86720
Nördlingen zurückzusenden, wobei die rechtzzitige Absendung genügt. Kosten und Gefahr der Rücksendung trägt der Empfänger.
hr Verla Ihr Verlag Franz Vahlen $\mathrm{GmbH}$, W

VERLAG VAHLEN · 80791 MÜNCHEN

Fax: (089) 381 89-402 - Internet: www.vahlen.de E-Mail: bestellung@vahlen.de

IV MARKETING · ZFP · 26. Jg. · Spezialausgabe „Dienstleistungsmarketing“ 2004 